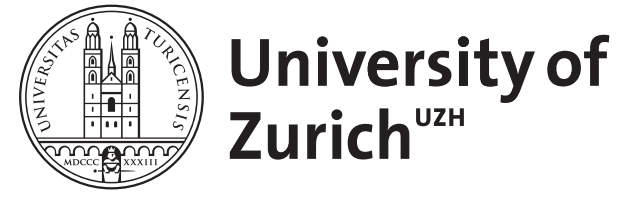

\title{
Models of Brauer-Severi surface bundles
}

Kresch, Andrew ; Tschinkel, Yuri

\begin{abstract}
We study Brauer-Severi surface bundles over smooth pro-jective varieties via root stacks, with a view towards applications tofailure of stable rationality.
\end{abstract}

DOI: https://doi.org/10.17323/1609-4514-2019-19-3-549-595

Posted at the Zurich Open Repository and Archive, University of Zurich ZORA URL: https://doi.org/10.5167/uzh-181594

Journal Article

Accepted Version

Originally published at:

Kresch, Andrew; Tschinkel, Yuri (2019). Models of Brauer-Severi surface bundles. Moscow Mathematical Journal, 19(3):549-595.

DOI: https://doi.org/10.17323/1609-4514-2019-19-3-549-595 


\title{
MODELS OF BRAUER-SEVERI SURFACE BUNDLES
}

\author{
ANDREW KRESCH AND YURI TSCHINKEL
}

\section{INTRODUCTION}

This paper is motivated by the study of rationality properties of Mori fiber spaces. These are algebraic varieties, naturally occurring in the minimal model program, typically via contractions along extremal rays; they are fibrations with geometrically rational generic fiber. Widely studied are conic bundles $\pi: X \rightarrow S$, when the generic fiber is a conic. According to the Sarkisov program [60], there exists a birational modification

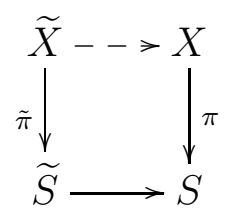

such that: (i) the general fiber of $\tilde{\pi}$ is a smooth conic, (ii) the discriminant divisor of $\tilde{\pi}$ is a simple normal crossing divisor, (iii) generally along the discriminant divisor, the fiber is a union of two lines, and (iv) over the singular locus of the discriminant divisor, the fiber is a double line in the plane.

Rationality of conic bundles over surfaces has been classically studied by Artin and Mumford [11], who produced examples of nonrational unirational threefolds of this type, computing their Brauer groups and using its nontriviality as an obstruction to rationality. This was generalized to higher-dimensional quadric bundles in [20], bringing higher unramified cohomology into the subject and providing further examples of nonrational varieties based on these new obstructions.

The specialization method, introduced by Voisin [64] and developed further by Colliot-Thélène-Pirutka [21], emerged as a powerful tool in the study of stable rationality. It allows to relate the failure of stable rationality of a very general member of a flat family to the existence of special fibers with nontrivial unramified cohomology and mild singularities; see also [13], [63]. In particular, the stable rationality problem for very general smooth rationally connected threefolds can be reduced to the case of conic bundles over rational surfaces [35], [42]. The case of very general families of conic bundles over rational surfaces is treated in [34].

Date: August 20, 2017. 
Many families that arise naturally in applications lead to discriminant curves of a special form. These have been studied, e.g., in [16] and [35].

A key step in the specialization method is the construction of families of Mori fiber spaces with controlled degeneration. A next case to consider is a fibration whose generic fiber is a form of $\mathbb{P}^{2}$.

Definition 1.1. Let $k$ be a perfect field of characteristic different from 3 and $\pi: X \rightarrow S$ a morphism of smooth projective varieties over $k$. We call $\pi$ a standard Brauer-Severi surface bundle if there exists a simple normal crossing divisor $D \subset S$ whose singular locus $D^{\text {sing }}$ is smooth, such that:

- $\pi$ is smooth over $S \backslash D$ and the generic fiber of $\pi$ is a nontrivial form of $\mathbb{P}^{2}$ over $k(S)$.

- Over every geometric point of $D \backslash D^{\text {sing }}$ the fiber of $\pi$ is a union of three Hirzebruch surfaces $\mathbb{F}_{1}$, meeting transversally, such that any pair of them meets along a fiber of one and the $(-1)$-curve of the other, while over the generic point of every irreducible component of $D$ the fiber of $\pi$ is irreducible.

- Over every geometric point of $D^{\text {sing }}$ the fiber of $\pi$ is an irreducible scheme whose underlying reduced subscheme is isomorphic to the cone over a twisted cubic curve.

We note that such $\pi: X \rightarrow S$ is necessarily flat. There is a geometric description, which in the case of algebraically closed base field is due to Artin [8], [9], and Maeda [47]:

Theorem 1.2. Let $k$ be a perfect field of characteristic different from 3 and $S$ a smooth projective algebraic variety over $k$. Assume that over $k$ the following holds: embedded resolution of singularities for reduced subschemes of $S$ of pure codimension 1, and desingularization for reduced finite-type schemes of pure dimension equal to $\operatorname{dim}(S)$ that is functorial with respect to étale morphisms. Let

$$
\pi: X \rightarrow S
$$

be a morphism of projective varieties over $k$, whose generic fiber is a nontrivial form of $\mathbb{P}^{2}$. Then there exists a commutative diagram

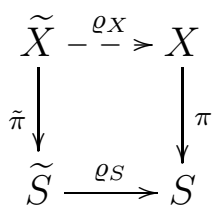

where if we let $U \subset S$ denote the locus over which $\pi$ is smooth,

- $\varrho_{S}$ is a birational morphism that restricts to an isomorphism over $U$, 
- $\varrho_{X}$ is a birational map that restricts to an isomorphism over $\varrho_{S}^{-1}(U) \rightarrow U$, and

- $\tilde{\pi}$ is a standard Brauer-Severi surface bundle.

Remark 1.3. When $\operatorname{dim}(S)=2$, only the embedded resolution for curves on $S$ is required for the proof of Theorem 1.2. However, functorial desingularization of surfaces is known even in positive characteristic [22]. We have decided not to mention the dimension of $S$ when stating the hypotheses concerning resolution of singularities.

In this paper we describe a technique based on root stacks, appearing in [34], that allows us to recover the Sarkisov program and its version by Artin and Maeda (Theorem 1.2) and obtain an extension to more general del Pezzo surface fibrations. The proof of Theorem 1.2 illustrates this technique, which leads to a global version of the constructions of Artin and Maeda that is crucial in moduli problems and in applications of specialization. As an application to rationality problems we prove:

Theorem 1.4. Let $S$ be a smooth del Pezzo surface of degree 2 over an uncountable algebraically closed field $k$ of characteristic $\neq 2,3$, and let $d \geq 3$. Then a standard Brauer-Severi surface bundle corresponding to a very general member of the linear system $\left|d\left(-K_{S}\right)\right|$ is not stably rational.

Acknowledgments: We are grateful to Brendan Hassett and Alena Pirutka for stimulating discussions on related topics and to Asher Auel for helpful comments. The first author is partially supported by the Swiss National Science Foundation. The second author is partially supported by NSF grant 1601912 .

\section{Preliminaries}

We start with some algebraic results that will be used in our approach. Let $k$ be a field. By a variety over $k$ we mean a geometrically integral separated finite-type scheme over $k$. We will need, however, the generality of locally Noetherian schemes and Deligne-Mumford stacks. This additional generality allows us to construct models of Brauer-Severi bundles in which all of the fibers are smooth. This comes at the cost of imposing nontrivial stack structure on the base.

2.1. Deligne-Mumford stacks. Deligne and Mumford [24] defined a class of stacks which includes all stack quotients of the form $[X / G]$, where $X$ is a variety and $G$ a finite group, and which are now called DeligneMumford stacks. The stack $[X / G]$ differs from the conventional quotient $X / G$ (which exists as a variety, e.g., when $X$ is quasi-projective) in that it keeps track of the stabilizers of the $G$-action. A further advantage is that 
$[X / G]$ has the same local properties (smoothness, etc.) as $X$. This will be crucial for us, as we will rely on properties of smooth varieties. Most important for us will be orbifolds over $k$, which are smooth geometrically integral separated finite-type Deligne-Mumford stacks over $k$ that possess an open substack isomorphic to a scheme.

As a stack, a scheme $X$ is encoded by the category of all schemes with morphism to $X$, and $[X / G]$, as $G$-torsors $E \rightarrow T$ with equivariant morphism $E \rightarrow X$. When $X$ is a point (i.e., $X=\operatorname{Spec}(k)$ if we work over a base field $k$, or $\operatorname{Spec}(\mathbb{Z})$ if we work with all schemes), $[X / G]$ is the category $B G$ of $G$-torsors, the classifying stack of $G$. We observe, the diagonal of $B G$ is a finite étale morphism of degree $|G|$.

An infinite discrete group $G$ is also permitted according to modern usage of the term Deligne-Mumford stack, leading to pathologies such as zero-dimensional Deligne-Mumford stack $\left[\operatorname{Spec}\left(\overline{\mathbb{Q}} \otimes_{\mathbb{Q}} \overline{\mathbb{Q}}\right) / H\right]$ for an index 2 non-open subgroup $H$ of $\operatorname{Gal}(\overline{\mathbb{Q}} / \mathbb{Q})$, which exists (by [61, Thm. 1]), is reduced (by $[29,4.3 .5]$ ), and is irreducible with 2 points (objects over $\operatorname{Spec}(\Omega)$ for all fields $\Omega$, modulo morphisms over $\operatorname{Spec}\left(\Omega^{\prime}\right) \rightarrow \operatorname{Spec}(\Omega)$ for embeddings $\Omega \rightarrow \Omega^{\prime}$ ).

When we restrict to Noetherian Deligne-Mumford stacks, i.e., quasicompact locally Noetherian Deligne-Mumford stacks with quasi-compact diagonal, such pathologies are excluded.

More general algebraic stacks such as $B G$ for positive-dimensional algebraic groups or nonreduced group schemes $G$ (Artin stacks) are not needed here.

2.2. Gerbes. Let $X$ be a Noetherian Deligne-Mumford stack and $n$ a positive integer, invertible in the local rings of an étale atlas of $X$. A gerbe over $X$ banded by roots of unity $\mu_{n}$, or just $\mu_{n}$-gerbe, is a DeligneMumford stack $G$ with morphism $G \rightarrow X$ that étale locally over $X$ is isomorphic to a product with the classifying stack $B \mu_{n}$ and is equipped with compatible identifications of the automorphism groups of local sections with $\mu_{n}$.

A $\mu_{n}$-gerbe is classified, up to isomorphism compatible with the identifications of automorphism groups of local sections, by a class in $H^{2}\left(X, \mu_{n}\right)$; cf. [50, §IV.2].

Vector bundles on a gerbe determine Brauer-Severi fibrations (see, e.g., [25]). This framework was essential in [34], in the construction of families of conic bundles for the application of the specialization method. We will use the same strategy for families of Brauer-Severi bundles of relative dimension 2 . 
2.3. Brauer groups. The Brauer group $\operatorname{Br}(K)$ of a field $K$ is a classical invariant, defined as the group of similarity classes of central simple algebras over $K$. It may also be described in terms of Galois cohomology, or in the language of étale cohomology, as

$$
\operatorname{Br}(K) \cong H^{2}\left(\operatorname{Spec}(K), \mathbb{G}_{m}\right) .
$$

(All cohomology in this paper will be étale cohomology.)

Now let $S$ be a Noetherian scheme or Deligne-Mumford stack. Similarity classes of sheaves of Azumaya algebras over $S$, which naturally generalize central simple algebras over a field, give one notion of Brauer group of $S$. Another, more relevant for us, is the cohomological Brauer group

$$
\operatorname{Br}(S):=H^{2}\left(S, \mathbb{G}_{m}\right)_{\text {tors }}
$$

The former is a subgroup of the latter; despite examples of a pathological nature where they differ [25] they are known to coincide in geometrically relevant cases, including all schemes that possess an ample line bundle; see, e.g., [45] and references therein.

Proposition 2.1 ([30, Cor. II.1.8, II.2.2], [34, Prop. 2]). Let $S$ be a regular integral Noetherian scheme or Deligne-Mumford stack and $S^{\prime} \subset S$ a nonempty open subscheme, respectively, substack. Then:

(i) The group $H^{2}\left(S, \mathbb{G}_{m}\right)$ is torsion, and the restriction homomorphism $\operatorname{Br}(S) \rightarrow \operatorname{Br}\left(S^{\prime}\right)$ is injective.

(ii) If $\operatorname{dim}(S) \leq 2$ then every element of $\operatorname{Br}(S)$ is the class of a sheaf of Azumaya algebras on $S$, and every sheaf of Azumaya algebras on $S^{\prime}$ representing an element $\left.\alpha\right|_{S^{\prime}}$ for some $\alpha \in \operatorname{Br}(S)$ extends to a sheaf of Azumaya algebras on $S$.

(iii) If $\operatorname{dim}(S)=2$ and $\operatorname{dim}\left(S \backslash S^{\prime}\right)=0$ then every sheaf of $A z u$ maya algebras on $S^{\prime}$ extends to a sheaf of Azumaya algebras on $S$, and the restriction homomorphism $\operatorname{Br}(S) \rightarrow \operatorname{Br}\left(S^{\prime}\right)$ is an isomorphism.

Now suppose $S$ is a smooth variety or orbifold over a field $k$, with function field $K=k(S)$. Then there is a description of the image of $\operatorname{Br}(S) \rightarrow \operatorname{Br}(K)$ in terms of residues. In case $S$ is an orbifold, the role of the residue field at a point is played by the residual gerbe

$$
\mathcal{G}_{\xi} \rightarrow \operatorname{Spec}(k(\xi))
$$

at a point $\xi$ of $S$ (cf. [43, $\S 11],[57$, App. B]).

Proposition 2.2. Let $k$ be a field, $S$ a smooth variety or orbifold over $k$ with function field $K$, and $n$ a positive integer that is invertible in $k$. Then there is a residue map from the $n$-torsion of the Brauer group of 
$K$ to the direct sum of $H^{1}$ groups of residual gerbes $\mathcal{G}_{\xi}$ at codimension 1 points $\xi \in S^{(1)}$, which fits into an exact sequence

$$
0 \rightarrow \operatorname{Br}(S)[n] \rightarrow \operatorname{Br}(K)[n] \rightarrow \bigoplus_{\xi \in S^{(1)}} H^{1}\left(\mathcal{G}_{\xi}, \mathbb{Z} / n \mathbb{Z}\right)
$$

Proof. We already know that $\operatorname{Br}(S) \rightarrow \operatorname{Br}(K)$ is injective. Cohomological purity $[10, \S$ XVI.3], combined with the local-to-global spectral sequence for cohomology with supports [50, §VI.5], yields exact sequences

$$
\begin{aligned}
\cdots \rightarrow H^{j-1} & (S \backslash T, \mathbb{Z} / n \mathbb{Z}(i)) \rightarrow H^{j-2 c}(T, \mathbb{Z} / n \mathbb{Z}(i-c)) \\
& \rightarrow H^{j}(S, \mathbb{Z} / n \mathbb{Z}(i)) \rightarrow H^{j}(S \backslash T, \mathbb{Z} / n \mathbb{Z}(i)) \rightarrow \ldots
\end{aligned}
$$

for all $i$ when $T \subset S$ is a closed substack of pure codimension $c$ that is also smooth over $k$. We may assume, as in [15, Rmk. 4.7], that $k$ is a perfect field, and as in [30] define the residue map and prove exactness.

Remark 2.3. When $S$ is a smooth variety the exact sequence is well known, cf. [19], and may be extended with a further residue map as a complex (Bloch-Ogus complex)

$$
\bigoplus_{\xi \in S^{(1)}} H^{1}(k(\xi), \mathbb{Z} / n \mathbb{Z}) \rightarrow \bigoplus_{\xi \in S^{(2)}} H^{0}\left(k(\xi), \mu_{n}^{-1}\right) .
$$

The complex with this additional residue map is exact when $k$ is algebraically closed and $S$ is a smooth projective rational surface.

2.4. Root stacks. We recall and describe basic properties of root stacks. Applied to a smooth variety and smooth divisor, the root stack construction produces an orbifold. The main function of a root stack is to remove ramification (e.g., of a Brauer class). In geometry, we find several advantages:

- We are able to relate smooth families over a root stack to flat families over the underlying scheme.

- The classification of smooth families may be more rigid, e.g., for conic bundles over a surface, the general ones correspond to Brauer classes at the generic point, while the smooth ones correspond to unramified Brauer classes.

- Other applications such as those requiring deformation theory also benefit from working with a proper Deligne-Mumford stack, instead of a quasi-projective variety.

Let $S$ be a regular integral scheme, $D \subset S$ an effective divisor, and $n$ a positive integer, invertible in the local rings of $S$. Then there is the corresponding root stack $\sqrt[n]{(S, D)}$, an integral locally Noetherian Deligne-Mumford stack, regular if and only if $D$ is regular $[18, \S 2],[1$, 
App. B]. Above $D$ is the gerbe of the root stack $\mathcal{G}_{D}$ [18, Def. 2.4.4], an effective divisor on $\sqrt[n]{(S, D)}$ fitting into a commutative diagram

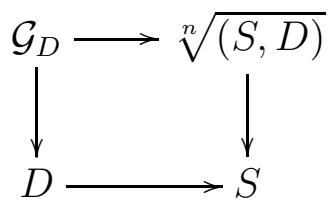

where the right-hand morphism is flat and is ramified over $D$ (so the diagram is not cartesian unless $D$ is empty) and restricts to an isomorphism over $S \backslash D$. The left-hand morphism makes $\mathcal{G}_{D}$ into a $\mu_{n}$-gerbe over $D$.

Remark 2.4. In the situation of Proposition 2.2 let $\xi$ be the generic point of an irreducible regular divisor $D$. The ramification of the right-hand map in (2.1) leads to a factor of $n$ in the residue map for the generic point of the gerbe of the root stack, as compared with that of $\xi$; cf. [59, Thm. 10.4]. So, as observed by Lieblich [46, §3.2], the residue map for the generic point of the gerbe of the root stack vanishes on $\operatorname{Br}(K)[n]$.

Now let $D^{\prime} \subset S$ be another effective divisor. The fiber product $\sqrt[n]{(S, D)} \times{ }_{S} \sqrt[n]{\left(S, D^{\prime}\right)}$ of the two associated root stacks is the iterated root stack $\sqrt[n]{\left(S,\left\{D, D^{\prime}\right\}\right)}\left[18\right.$, Def. 2.2.4]. If $D$ and $D^{\prime}$ are regular and intersect transversally, then $\sqrt[n]{\left(S,\left\{D, D^{\prime}\right\}\right)}$ is regular; the same is valid with any number of divisors. A generalization (not used in this paper) is the case of a divisor $D$ that étale locally may be written as a union of regular divisors meeting transversally, for which a construction by Matsuki and Olsson [48] based on log structures gives rise to a regular DeligneMumford stack, étale locally isomorphic to an iterated root stack.

Locally on $S$ the divisor $D$ is defined by the vanishing of a regular function $f$, and then $\sqrt[n]{(S, D)}$ is the quotient stack of $\operatorname{Spec}\left(\mathcal{O}_{S}[t] /\left(t^{n}-f\right)\right)$ by the scalar action of $\mu_{n}$ on $t$. The gerbe of the root stack is, locally, defined by the vanishing of $t$, and its points are just the points of $D$, with $\mu_{n}$-stabilizer, i.e., above any point $x \in D$ with residue field $\kappa(x)$ the gerbe of the root stack takes the form of a classifying stack $B G$ for $G=\mu_{n, \kappa(x)}$.

Often we consider flat families of projective varieties, or schemes, and we benefit by being able to study such families over a root stack, i.e., flat projective morphisms $P \rightarrow \sqrt[n]{(S, D)}$ of Deligne-Mumford stacks. The fiber over a point $s \in D$, where the gerbe of the root stack takes the form $B G$ for $G=\mu_{n, \kappa(s)}$ as mentioned above, has the form $\left[P_{s} / G\right]$ for an action of $G$ on a projective scheme $P_{s}$ over $\kappa(s)$. Typically $G$ acts with stabilizers $\left(\mu_{n}\right.$ or subgroups of $\left.\mu_{n}\right)$, and we will speak of the locus with $\mu_{n}$-stabilizer for the appropriate closed subscheme of $P_{s}$. 
2.5. Coarse moduli spaces. Let $X$ be a Deligne-Mumford stack, and assume that $X$ has finite stabilizer, meaning that projection to $X$ from $X \times_{X \times X} X$ is a finite morphism. Then there is a coarse moduli space, an algebraic space $Q$ with separated morphism $X \rightarrow Q$, universal for morphisms to algebraic spaces. For instance, $X$ could be $[\operatorname{Spec}(A) / G]$ for some finite group $G$ acting on affine scheme $\operatorname{Spec}(A)$, and then $X$ has coarse moduli space $\operatorname{Spec}\left(A^{G}\right)$, where $A^{G}$ denotes the $G$-invariant subring of $A$. The article by Keel and Mori [38] gives the classic treatment of coarse moduli space in the setting where $X$ is locally of finite type over a locally Noetherian base scheme; this suffices for our purposes, and in this case $Q$ has the same property and $X \rightarrow Q$ is proper and quasifinite. The treatment without finiteness hypothesis is due to Rydh [58]. (These treatments allow, more generally, $X$ to be an Artin stack with finite stabilizer.)

The construction of coarse moduli space shows that $X \rightarrow Q$ has the étale local (over $Q$ ) form $[\operatorname{Spec}(A) / G] \rightarrow \operatorname{Spec}\left(A^{G}\right)$, as described above. In fact, $G$ may be taken to be the geometric stabilizer group at a point of $X$, and the assertion is valid on an étale neighborhood of the corresponding point of $Q$; cf. [5, Lem. 3.4].

For the next statement we assume that $X$ is tame, meaning that besides the finite stabilizer hypothesis we also require the order of the geometric stabilizer group at any point $\operatorname{Spec}(\Omega) \rightarrow X$ not to be divisible by the characteristic of $\Omega$ [56]. (This notion is also available for Artin stacks with finite stabilizer [2].) We suppose furthermore that $X$, and hence as well $Q$, is Noetherian. Then by [3, Thm. 10.3], pullback by $X \rightarrow Q$ identifies the category of vector bundles on $Q$ with the full subcategory of vector bundles on $X$ with trivial actions of geometric stabilizer groups at closed points.

The most relevant example of coarse moduli space for us is a root stack $\sqrt[n]{(S, D)} \rightarrow S$ (or an iterated root stack), and this is tame. The next result concerns schemes, Fano over $S$ for a Noetherian scheme $S$, by which we mean the full subcategory of schemes over $S$, in which an object is a flat projective morphism $Z \rightarrow S$ with Gorenstein fibers such that $\omega_{Z / S}^{\vee}$ is relatively ample. The last condition is equivalent by $[28,4.7 .1]$ to the ampleness of $\omega_{Z_{s}}^{\vee}$ on the fiber $Z_{s}$ for every closed point $s \in S$. We may also take $S$ to be an algebraic space, in which case $Z$ is also allowed to be an algebraic space. We may allow $S$ to be a DM stack, in which case $Z$ is also a DM stack. But since stacks form a 2-category, we need to take care to obtain an ordinary category: a morphism from $f: Z \rightarrow S$ to $f^{\prime}: Z^{\prime} \rightarrow S$ is defined to be an equivalence class of pairs $(\varphi, \alpha)$ where $\varphi: Z \rightarrow Z^{\prime}$ is a morphism and $\alpha: f \Rightarrow f^{\prime} \circ \varphi$ is a 2-morphism, 
with $(\varphi, \alpha) \sim(\tilde{\varphi}, \tilde{\alpha})$ when there exists a (necessarily unique) 2-morphism $\beta: \varphi \Rightarrow \tilde{\varphi}$, such that $f^{\prime}(\beta(z)) \circ \alpha(z)=\tilde{\alpha}(z)$ for every object $z$ of $Z$.

Proposition 2.5. Let $X$ be a tame Noetherian DM stack with coarse moduli space $X \rightarrow Q$. Then the category of schemes, Fano over $Q$, is equivalent, by base change, to the full subcategory of DM stacks, Fano over $X$, with trivial actions of geometric stabilizer groups at closed points.

Proof. The universal property of the coarse moduli space tells us that base change by $X \rightarrow Q$ is a fully faithful functor.

To an object $f: Z \rightarrow X$ of the category of DM stacks, Fano over $X$, there is an associated quasi-coherent sheaf of graded $\mathcal{O}_{X}$-algebras $\bigoplus_{n \geq 0} f_{*}\left(\left(\omega_{Z / X}^{\vee}\right)^{n}\right)$. The graded components are coherent and for sufficiently large $n$ are locally free with formation commuting with base change to (geometric) fibers. If the actions of geometric stabilizer groups at closed points is trivial, then $f_{*}\left(\left(\omega_{Z / X}^{\vee}\right)^{n}\right)$ for $n \gg 0$ satisfies the condition stated above to descend to a locally free coherent sheaf on $Q$. The algebra structure descends as well, and by applying Proj we obtain an algebraic space $Y$, Fano over $Q$, which upon base change to $X$ recovers $Z \rightarrow X$ up to an isomorphism.

Remark 2.6. As a consequence, the algebraic space $Y$, Fano over $Q$ that we obtain in the proof of Proposition 2.5, is the coarse moduli space of $Z$.

2.6. Étale local uniqueness in smooth families. In certain geometric situations such as fibrations in projective spaces, a smooth family has a unique étale local isomorphism type. The same holds $G$-equivariantly, when $G$ is a finite group whose order is not divisible by the residue characteristic.

Lemma 2.7. Let $A$ be a Henselian ring with residue field $\kappa$, and $X$ a scheme, smooth over $\operatorname{Spec}(A)$. Let $G$ be a finite group, whose order is invertible in $\kappa$, with compatible actions on $X$ and on $A$ and trivial action on $\kappa$, and $x$ a $\kappa$-point of the fiber $X_{\kappa}$ over the closed point of $\operatorname{Spec}(A)$ that is fixed for the group action. Then $X$ admits a $G$-invariant A-point that specializes to $x$.

Proof. Since $A$ and hence as well $A^{G}$ is Henselian, there is a free finiterank $A^{G}$-module with $G$-action, whose base change to $\kappa$ is isomorphic to any given finite-dimensional $\kappa$-vector space with $G$-action.

Replacing, as needed, $X$ by intersections of translates under $G$ of an affine neighborhood of $x$, we may suppose that $X=\operatorname{Spec}(B)$ is affine. Let $\mathfrak{m}$ denote the maximal ideal corresponding to $x$. The $A$-linear map

$$
\mathfrak{m} \rightarrow \Omega_{X_{\kappa}, x}^{1}
$$


(sending $f$ to $d f$ ) is surjective and $G$-equivariant. There is therefore a lift of an appropriate $\kappa$-basis of $\Omega_{X_{\kappa}, x}^{1}$ to elements of $\mathfrak{m}$ whose $A^{G}$-linear span is invariant under the $G$-action. According to $[29,17.16 .3(\mathrm{i})]$ (really, its proof), the lifts define a $G$-invariant subscheme $W \subset X$ that is étale over $\operatorname{Spec}(A)$ at $x$. Since $A$ is Henselian, there is a unique $G$-invariant $A$-point of $W$ that specializes to $x$.

Lemma 2.8. Let $A$ be a Henselian ring with residue field $\kappa$, and $X$ and $Y$ schemes, smooth and projective over $\operatorname{Spec}(A)$. Let $G$ be a finite group, whose order is invertible in $\kappa$, with compatible actions on $X$, on $Y$, and on $A$, with trivial action on $\kappa$. Let $\varphi: X_{\kappa} \rightarrow Y_{\kappa}$ be a G-equivariant isomorphism of fibers. If the tangent bundle $T_{X_{\kappa}}$ satisfies

$$
H^{1}\left(X_{\kappa}, T_{X_{\kappa}}\right)=0,
$$

then there exists a $G$-equivariant isomorphism $X \rightarrow Y$ over $\operatorname{Spec}(A)$ that specializes to $\varphi$.

Proof. There is a scheme $\operatorname{Isom}_{A}(X, Y)$, parametrizing isomorphisms of fibers $[31, \S 4 \mathrm{c}]$, with $G$-action, $\operatorname{smooth}$ over $\operatorname{Spec}(A)$ at $\varphi$ by $[31$, Cor. 5.4]. We conclude by Lemma 2.7 .

2.7. Components in families. The irreducible components of fibers in a flat family with geometrically reduced fibers may be accessed by excluding the relative singular locus and forming the sheaf of connected components. If $\pi: X \rightarrow S$ is a finitely presented smooth morphism of schemes, then geometric components of fibers determine an étale equivalence relation on $X$ over $S$ whose algebraic space quotient $\pi_{0}(X / S)$ is étale over $S$ and is characterized as factorizing $\pi$,

$$
X \rightarrow \pi_{0}(X / S) \rightarrow S
$$

such that $X \rightarrow \pi_{0}(X / S)$ is surjective with geometrically connected fibers $[43,6.8]$. There are easy examples for which $\pi_{0}(X / S)$ is not a scheme, e.g., the sum of squares of coordinates $\mathbb{A}_{\mathbb{R}}^{2} \backslash\{(0,0)\} \rightarrow \mathbb{A}_{\mathbb{R}}^{1}$. Formation of $\pi_{0}(X / S)$ commutes with base change by an arbitrary morphism $S^{\prime} \rightarrow S$.

A subset of $X$, which is the pre-image of a subset of $\pi_{0}(X / S)$, will be called component-saturated.

Lemma 2.9. Let $\pi: X \rightarrow S$ be a proper flat finitely presented puredimensional morphism of schemes, such that for every $s \in S$ the smooth locus of the fiber $X_{s}$ is dense in $X_{s}$. Then the morphism $\pi_{0}\left(X^{\mathrm{sm}} / S\right) \rightarrow S$ is universally closed, where $X^{\mathrm{sm}}$ denotes the relative smooth locus of $\pi$. If, moreover, the geometric fibers of $\pi$ all have $d$ irreducible components for some integer $d$, then $\pi_{0}\left(X^{\mathrm{sm}} / S\right) \rightarrow S$ is finite étale of degree $d$. 
Proof. The second assertion follows from the first by the general fact that a finitely presented universally closed étale morphism $T \rightarrow S$ with fibers all of the same degree is finite. This comes down to topological properties of the relative diagonal (it is an open immersion since $T \rightarrow S$ is étale, and we need to show that it is closed as well), so the verification is reduced by $[29,1.10 .1]$ to the case that $S$ is Spec of a local ring, and by $[29,2.6 .2]$, to the case of a strictly Henselian ring. Each of the $d$ pre-images of the closed point then lifts to a section. The sections cover $T$, since the image of their complement is closed but does not contain the closed point of $S$. So, $T$ is the disjoint union of the sections.

Now suppose that $S=\operatorname{Spec}(A)$, where $A$ is a local ring. Let $z \in X$ be a maximal point of a fiber of $\pi$ and $Z$ its closure in $X$. We claim that $\pi\left(Z \cap X^{\mathrm{sm}}\right)$ contains the closed point of $S$. Indeed, by semicontinuity of fiber dimension [29, 13.1.5], $Z$ contains a maximal point of the fiber over the closed point, which by hypothesis lies in $X^{\mathrm{sm}}$.

Let $n \geq 0$. In a proper flat family of schemes of relative dimension $n$ over a Noetherian base scheme, the condition on fibers to have singular locus of dimension less than $n$ is open by semicontinuity of fiber dimension. With the local form of semicontinuity of fiber dimension $[29,13.1 .3]$ (semicontinuity of $w \mapsto \min \operatorname{dim}\left(\pi^{-1}(\pi(w)) \cap U\right)$ where the minimum is over open $U$ containing $w$ ) and flatness (particularly, [29, 2.3.4]), we see that the condition to have pure dimension $n$ is closed. We reduce the verification of the first assertion immediately to the case when $S$ is affine and reduced, and with the preceding observations and $[29,8.10 .5,11.2 .7]$ to the case when $S$ is Noetherian. Then, as noted in the proof of [41, Prop. 2.2], the claim implies the assertion. (In fact, the case $A$ is a DVR suffices.)

Remark 2.10. With the notation of Lemma 2.9, any component-saturated closed subset $W \subset X^{\mathrm{sm}}$ has the property that formation of the closure $\bar{W}$ in $X$ commutes with base change by an arbitrary morphism $S^{\prime} \rightarrow S$. Since an equality of sets may be verified fiberwise, the assertion reduces to the case $S^{\prime}=\operatorname{Spec}(\kappa(s))$, where $\kappa(s)$ is the residue field of a point $s \in S$. We may suppose $\pi$ has constant fiber dimension $n$. For $x \in \bar{W} \cap \pi^{-1}(s)$, semicontinuity of fiber dimension yields $\operatorname{dim}\left(\pi^{-1}(s) \cap U\right)=n$ for all open $U \subset \bar{W}$ containing $x$. Hence there is a maximal point of $\pi^{-1}(s)$ that lies in $W$ (equivalently, lies in $\bar{W}$ ) and specializes to $x$.

\section{Conic Bundles}

In this section we present an ingredient from the Sarkisov program, which gives a description of conic bundles with regular discriminant divisor, using the language of root stacks. 
By a conic bundle over a locally Noetherian scheme $S$, in which 2 is invertible in the local rings, we mean a flat projective morphism $\pi: X \rightarrow S$ such that the fiber over every point of $S$ is isomorphic to a possibly singular, possibly non-reduced conic in $\mathbb{P}^{2}$, i.e., the zero-loci of a nontrivial ternary quadratic form. We say that a conic bundle $\pi: X \rightarrow S$ has mild degeneration if all fibers are reduced.

Proposition 3.1. Let $S$ be a regular scheme, such that 2 is invertible in the local rings of $S$, and let $D \subset S$ be a regular divisor. Then the operations described below identify, up to unique isomorphism:

- smooth $\mathbb{P}^{1}$-fibrations over $\sqrt{(S, D)}$ having nontrivial $\mu_{2}$-actions on fibers over the gerbe of the root stack, with

- conic bundles $\pi: X \rightarrow S$ such that $X$ is regular and the fiber $\pi^{-1}(s)$ over a point $s \in S$ is singular if and only if $s \in D$; all such conic bundles have mild degeneration.

Given a smooth $\mathbb{P}^{1}$-fibration $P \rightarrow \sqrt{(S, D)}$ with nontrivial $\mu_{2}$-actions on fibers over the gerbe of the root stack, a conic bundle $\pi: X \rightarrow S$ with mild degeneration is obtained by

- blowing up the locus with $\mu_{2}$-stabilizer,

- collapsing the "middle components" of fibers over D, and

- descending to $S$.

Given a conic bundle $\pi: X \rightarrow S$ we obtain the associated smooth $\mathbb{P}^{1}$ fibration over $\sqrt{(S, D)}$ (up to unique isomorphism) by

- pulling back to $\sqrt{(S, D)}$,

- blowing up the relative singular locus over the gerbe of the root stack, and

- collapsing the "end components" of fibers over D.

Proposition 3.1 deals with flat projective families of reduced curves with at most nodes as singularities. This is the setting of (pre)stable curves, for which there are known constructions to perform the contractions indicated in the statement of Proposition 3.1. Collapsing the "middle components" of a flat family of at-most-3-component genus 0 prestable curves $\pi: C \rightarrow T$ is achieved by the relative anticanonical model Proj $\left(\bigoplus_{m \geq 0} \pi_{*}\left(\left(\omega_{C / T}^{\vee}\right)^{m}\right)\right)$. The setting where "end components" are to be collapsed is one of families with only 1- and 3-component genus 0 prestable curves, and étale locally there exists a section $\sigma$ which avoids the end components of 3 -component fibers; then Proj $\left(\bigoplus_{m>0} \pi_{*}\left(\mathcal{O}_{C}(\sigma)^{m}\right)\right)$ gives the desired contraction, and although sections exist only étale locally, the construction may be performed globally over the base, as described in [41]. 
Proof. Let $P \rightarrow \sqrt{(S, D)}$ be a smooth $\mathbb{P}^{1}$-fibration, such that $\mu_{2}$ acts nontrivially on $P_{s}$ for every $s \in D$ in the notation from the discussion of stabilizers from $\S 2.4$. Let $\operatorname{Spec}(A)$ be an affine neighborhood in $S$ of a point $s \in D$, on which $D$ is defined by the vanishing of a function $f \in A$. Setting

$$
A^{\prime}:=A[t] /\left(t^{2}-f\right),
$$

then, we have an open substack of $\sqrt{(S, D)}$ isomorphic to

$$
\left[\operatorname{Spec}\left(A^{\prime}\right) / \mu_{2}\right] \text {, }
$$

where $\mu_{2}$ acts by scalar multiplication on $t$. We claim that there is an étale neighborhood $\operatorname{Spec}(B) \rightarrow \operatorname{Spec}(A)$ of $s$ such that for the rank 2 vector bundle and projectivization

$$
V:=\left[\operatorname{Spec}\left(A^{\prime}[u, v]\right) / \mu_{2}\right] \quad \text { and } \quad \widehat{P}:=\mathbb{P}(V),
$$

where $\mu_{2}$ acts by scalar multiplication on $u$ and trivially on $v$, we have

$$
\operatorname{Spec}(B) \times_{S} P \cong \operatorname{Spec}(B) \times{ }_{S} \widehat{P} .
$$

Equivalently,

$$
\operatorname{Spec}\left(A^{s h}\right) \times{ }_{S} P \cong \operatorname{Spec}\left(A^{s h}\right) \times{ }_{S} \widehat{P},
$$

where $A^{\text {sh }}$ is a strict Henselization of the local ring of $\operatorname{Spec}(A)$ at $s$. By $[29,18.8 .10]$, we have

$$
A^{s h} \otimes_{A} A^{\prime} \cong A^{\prime s h},
$$

where $A^{\prime s h}$ is an analogous strict Henselization of $A^{\prime}$. So, it suffices to verify that

$$
\operatorname{Spec}\left(A^{\prime s h}\right) \times \sqrt{(S, D)} P \quad \text { and } \quad \operatorname{Spec}\left(A^{\prime s h}\right) \times \sqrt{(S, D)} \widehat{P}
$$

are equivariantly isomorphic. By Lemma 2.8 it is enough to check that the respective fibers over the closed point of $\operatorname{Spec}\left(A^{\prime s h}\right)$ are equivariantly isomorphic, and this is so by the hypothesis of nontrivial $\mu_{2}$-action on fibers of $P$ over the gerbe of the root stack. In particular, the stabilizer locus of $P$ is a degree 2 étale representable cover of the gerbe of the root stack.

The blow-up $P_{1}$ of the locus of $P$ with $\mu_{2}$-stabilizer therefore has geometric fibers over points of $D$ with three irreducible components and nontrivial $\mu_{2}$-action only on the "middle components". After collapsing "middle components" to $P_{2}$, the geometric fibers have two irreducible components over points of $D$ and trivial $\mu_{2}$-action. By Proposition 2.5, $P_{2} \rightarrow \sqrt{(S, D)}$ descends to a conic bundle $P_{3} \rightarrow S$. To see that $P_{1}$ is the blow-up of $P_{2}$ along the relative singular locus over the gerbe of the root stack, we use the fact that the constructions described in the statement 
are compatible with base change by an étale morphism to $S$ and carry out the constructions with $\widehat{P}$ :

$$
\begin{aligned}
& \widehat{P}_{1}=\left[\operatorname{Proj}\left(A^{\prime}[p, q, r, w] /\left(p r-t q^{2}, p w-t^{2} q r, q w-t r^{2}\right)\right) / \mu_{2}\right], \\
& \widehat{P}_{2}=\left[\operatorname{Proj}\left(A^{\prime}[x, y, z] /\left(x z-t^{2} y^{2}\right)\right) / \mu_{2}\right], \\
& \widehat{P}_{3}=\operatorname{Proj}\left(A[x, y, z] /\left(x z-f y^{2}\right)\right) .
\end{aligned}
$$

Since $\widehat{P}_{3}$ is regular, so is $P_{3}$. The steps, performed in reverse starting from $P_{3}$, yield $P$ up to unique isomorphism.

A conic bundle $X \rightarrow S$ with $X$ regular and singular fibers precisely over points of $D$ has, locally, defining equation $f x^{2}+a y^{2}+b z^{2}=0$ for units $a$ and $b$, as we see using diagonalizability of a quadratic form over $\mathcal{O}_{S, s}$, first with $s$ the generic point of a component of $D$ and then with arbitrary $s \in D$. (Such an observation for quadric bundles is given in [12, Prop. 1.2.5].) So, étale locally, there is an isomorphism with $\widehat{P}_{3}$, and the construction may be carried out to yield a smooth $\mathbb{P}^{1}$-bundle over $\sqrt{(S, D)}$. From this, the construction of blowing up, collapsing, and descending to $S$ recovers $X \rightarrow S$ up to unique isomorphism.

Remark 3.2. The Sarkisov program establishes the existence of good models of conic bundles over arbitrary varieties [60], via classical birational geometry. A more general version of this result may be obtained through the use of root stacks. Details are presented in [55].

\section{Brauer-SEVEri SURFACE BUNDles}

In the following sections we prove Theorem 1.2 using root stacks.

Definition 4.1. Let $S$ be a locally Noetherian scheme, in which 3 is invertible in the local rings. A Brauer-Severi surface bundle over $S$ is a flat projective morphism $\pi: X \rightarrow S$ such that the fiber over every geometric point of $S$ is isomorphic to one of the following:

- a del Pezzo surface of degree 9 (i.e., since a geometric fiber, $\mathbb{P}^{2}$ ),

- the union of three Hirzebruch surfaces $\mathbb{F}_{1}$, meeting transversally, such that any pair of them meets along a fiber of one and the $(-1)$-curve of the other,

- an irreducible scheme whose underlying reduced subscheme is isomorphic to the cone over a twisted cubic curve.

If only fibers of the first two types appear, then we say that the BrauerSeveri surface bundle has mild degeneration.

Remark 4.2. For the fibers $X_{s}$ appearing in a Brauer-Severi surface bun-

dle with mild degeneration, the dual $\omega_{X_{s}}^{\vee}$ of the dualizing sheaf is very 
ample and we have $h^{0}\left(X_{s}, \omega_{X_{s}}^{\vee}\right)=10$. This is standard for del Pezzo surfaces of degree 9 and may be verified with a short computation for unions of three Hirzebruch surfaces.

Definition 4.3. Let $k$ be a field of characteristic different from 3. An action of $\mu_{3}$ on $\mathbb{P}_{k}^{2}$ is said to be balanced if, after passing to an algebraic closure $\bar{k}$ of $k$ and making a change of projective coordinates, the action is $\mu_{3} \times \mathbb{P}_{\bar{k}}^{2} \rightarrow \mathbb{P}_{\bar{k}}^{2}$,

$$
(\alpha, x: y: z) \mapsto\left(\alpha x: \alpha^{2} y: z\right) .
$$

We formulate an analogue of Proposition 3.1.

Proposition 4.4. Let $S$ be a regular scheme, such that 3 is invertible in the local rings of $S$, and let $D \subset S$ be a regular divisor. Then the operations described below identify, up to unique isomorphism:

- smooth $\mathbb{P}^{2}$-fibrations over $\sqrt[3]{(S, D)}$ having balanced $\mu_{3}$-actions on fibers over the gerbe of the root stack, with

- Brauer-Severi surface bundles $\pi: X \rightarrow S$ such that $X$ is regular and the fiber $\pi^{-1}(s)$ over a point $s \in S$ is singular if and only if $s \in D$; all such bundles have mild degeneration.

Given a smooth $\mathbb{P}^{2}$-fibration $P \rightarrow \sqrt[3]{(S, D)}$ with balanced $\mu_{3}$-actions on fibers over the gerbe of the root stack, $P$ has distinguished loci

- $P_{\mu}$ : locus with nontrivial $\mu_{3}$-stabilizer,

- $P_{\lambda}$ : union of lines on the geometric $\mathbb{P}^{2}$-fibers joining points of $P_{\mu}$.

On the blow-up $B \ell_{P_{\mu}} P$ of $P$ we have a distinguished locus

- $P_{c}$ : the union of codimension 2 components of the stabilizer locus.

On the second blowup $B \ell_{P_{c}}\left(B \ell_{P_{\mu}} P\right)$ we have the locus

- $\widetilde{P}_{\lambda}$ : the proper transform of $P_{\lambda}$.

Then a Brauer-Severi surface bundle $\pi: X \rightarrow S$ with mild degeneration results by

- collapsing first the degree 8 components of fibers over $D$ of

$$
B \ell_{\widetilde{P}_{\lambda}}\left(B \ell_{P_{c}}\left(B \ell_{P_{\mu}} P\right)\right)
$$

- collapsing the degree 3 components,

- descending to $S$.

Given a Brauer-Severi surface bundle $\pi: X \rightarrow S$ we obtain the associated smooth $\mathbb{P}^{2}$-fibration over the root stack $\sqrt[3]{(S, D)}$ (up to unique isomorphism) by

- pulling back to $\sqrt[3]{(S, D)}$,

- blowing up the relative singular locus of the relative singular locus over the gerbe of the root stack, 
- blowing up the relative singular locus over the gerbe of the root stack, and

- contracting the high-degree $(\geq 7)$ components above the gerbe of the root stack.

The constructions in Proposition 4.4 are more complicated than the corresponding constructions for conic bundles. Their justification relies on machinery presented in an Appendix on birational contractions.

The proof, given in the following sections, has several ingredients:

- the forwards construction, which produces a mildly degenerating Brauer-Severi surface bundle out of a smooth of $\mathbb{P}^{2}$-fibration over the root stack, is carried out using explicit birational contractions (Section 5);

- the reverse construction, starting with a mildly degenerating BrauerSeveri surface bundle, is carried out with similiar techniques (Section 6);

- the verification that every Brauer-Severi surface bundle $\pi: X \rightarrow$ $S$, such that $X$ is regular and precisely the fibers over a regular divisor on $S$ are singular, has mild degeneration, based on a combination of the forwards construction and purity for the Brauer group (Section 7);

\section{Forwards CONSTRUCTION}

In this section we carry out the forwards construction of Proposition 4.4 .

Let $P \rightarrow \sqrt[3]{(S, D)}$ be a smooth $\mathbb{P}^{2}$-fibration, such that the $\mu_{3}$-action on $P_{s}$ for every $s \in D$ is balanced. As in the proof of Proposition 3.1, there is an affine étale neighborhood $\operatorname{Spec}(B) \rightarrow S$ of a point $s \in D$, where the pre-image of $D$ is principal, say, defined by the vanishing of $f \in B$, and setting

$$
B^{\prime}:=B[t] /\left(t^{3}-f\right), \quad V:=\left[\operatorname{Spec}\left(B^{\prime}[u, v, w]\right) / \mu_{3}\right], \quad \widehat{P}:=\mathbb{P}(V),
$$

we have an isomorphism

$$
\operatorname{Spec}(B) \times_{S} P \cong \widehat{P} .
$$

Here, $\mu_{3}$ acts on by scalar multiplication on $t$ and $u$, by scalar multiplication squared on $v$, and trivially on $w$. In particular, $P_{\mu}$ is a degree 3 étale representable cover of the gerbe of the root stack, and after blowing up $P_{c}$ is smooth over the gerbe of the root stack, consisting of a line in every $\mathbb{P}^{2}$ fiber of the exceptional divisor, and $\widetilde{P}_{\lambda}$ is also smooth over the gerbe of the root stack, consisting geometrically of three pairwise disjoint $(-2)$-curves in every component of fibers over the gerbe of the root stack, 
isomorphic to the minimal resolution of the singular cubic surface of type $3 \mathrm{~A}_{2}[17]$.

At the successive blow-ups we have divisors at our disposal as described below; for the description of combinations that are relatively ample over $S$ we use the fact about ampleness mentioned in $\S 2.5$ in combination with the equivalence $[28,2.6 .2]$ of ampleness of a line bundle on a projective scheme with ampleness of its restriction to every irreducible component, to characterize relatively ample line bundles. We adopt the convention that the same notation will be used for a divisor or line bundle and its pullbacks under the morphisms described below. But in this section, tilde $(\sim)$, as in $\widetilde{E}_{1}$, always indicates proper transform. We let $\omega^{\vee}$ denote the relative anticanonical bundle of $P$ over $\sqrt[3]{(S, D)}$.

- We analyze the first blow-up

$$
B \ell_{P_{\mu}} P \rightarrow P \text {. }
$$

In addition to $\omega^{\vee}$, we have the exceptional divisor $E_{1}$; on geometric fibers over the gerbe of the root stack, $E_{1}$ consists of three components $E_{u}, E_{v}, E_{w}$, each isomorphic to $\mathbb{P}^{2}$, attached along three pairwise disjoint exceptional divisors $d_{u}, d_{v}, d_{w}$ of a component $\Sigma_{1}$ isomorphic to a degree 6 del Pezzo surface (DP6), with the remaining exceptional divisors $\lambda_{u v}, \lambda_{v w}, \lambda_{u w} \subset \Sigma_{1}$ contained in the proper transform of $P_{\lambda}$. The intersection numbers are:

\begin{tabular}{c|cc} 
& $d_{u}$ & $\lambda_{v w}$ \\
\hline$\omega^{\vee}$ & 0 & 3 \\
$E_{1}$ & -1 & 2
\end{tabular}

The projection formula supplies the first row. Entries in the second row are intersection numbers on DP6 with $d_{u}+d_{v}+d_{w}$.

Multiples of $\alpha \omega^{\vee}-E_{1}$ for $\alpha \in \mathbb{Q}, \alpha>2 / 3$ are relatively ample.

- We analyze the second blowup

$$
B \ell_{P_{c}}\left(B \ell_{P_{\mu}} P\right) \rightarrow B \ell_{P_{\mu}} P .
$$

Let $E_{2}$ be its exceptional divisor. With notation as above and components $\ell_{u} \subset E_{u}$, etc., of the stabilizer locus $P_{c}$, we have the exact sequence of normal bundles

$$
\left.\left.\left.0 \rightarrow N_{P_{c} / E_{1}}\right|_{\ell_{u}} \rightarrow N_{P_{c} / B \ell_{P_{\mu}} P}\right|_{\ell_{u}} \rightarrow N_{E_{1} / B \ell_{P_{\mu}} P}\right|_{\ell_{u}} \rightarrow 0
$$

with

$$
\operatorname{deg}\left(N_{\ell_{u} / E_{u}}\right)=1 \quad \text { and } \quad \operatorname{deg}\left(\left.N_{E_{u} / B \ell_{P_{\mu}} P}\right|_{\ell_{u}}\right)=-1
$$

(and similarly for $\ell_{v}$ and $\ell_{w}$ ). On geometric fibers over the gerbe of the root stack, $E_{2}$ consists of three components $F_{u}, F_{v}, F_{w}$, each isomorphic to a Hirzebruch surface $\mathbb{F}_{2}$, glued along (-2)-curves 
$c_{u}$, respectively, $c_{v}, c_{w}$, to $\widetilde{E}_{u}$, respectively, $\widetilde{E}_{v}, \widetilde{E}_{w}$, and along a fiber $f_{2, u}$ (respectively, $f_{2, v}, f_{2, w}$ ) to $\Sigma_{2}$, the blow-up of $\Sigma_{1}$ at three points; note that $\Sigma_{2}$ is isomorphic to the minimal resolution of the singular cubic surface of type $3 \mathrm{~A}_{2}$. The class of the exceptional divisor $E_{2}$, restricted to $F_{u}$, etc., is $\mathcal{O}_{\mathbb{P}\left(\mathcal{O}_{\mathbb{P} 1}(1) \oplus \mathcal{O}_{\mathbb{P} 1}(-1)\right)}(-1)$. The further restriction to $c_{u}$, etc., is $\mathcal{O}_{\mathbb{P}\left(\mathcal{O}_{\mathbb{P} 1}(1) \oplus 0\right)}(-1) \cong \mathcal{O}_{\mathbb{P}^{1}}(1)$ and to $f_{2, u}$, etc., is isomorphic to $\mathcal{O}_{\mathbb{P}^{1}}(-1)$; these explain the last two entries in the third row:

\begin{tabular}{c|cccc} 
& $\tilde{d}_{u}$ & $\tilde{\lambda}_{v w}$ & $c_{u}$ & $f_{2, u}$ \\
\hline$\omega^{\vee}$ & 0 & 3 & 0 & 0 \\
$E_{1}$ & -1 & 2 & -1 & 0 \\
$E_{2}$ & 1 & 1 & 1 & -1
\end{tabular}

Intersection numbers on $\Sigma_{2}$ give the first two entries in the third row.

Multiples of $\beta\left(\alpha \omega^{\vee}-E_{1}\right)-E_{2}$ with

$$
\alpha>(1 / 3)(2+1 / \beta) \quad \text { and } \quad \beta>1
$$

are relatively ample.

- We analyze the third blowup

$$
B \ell_{\widetilde{P}_{\lambda}}\left(B \ell_{P_{c}}\left(B \ell_{P_{\mu}} P\right)\right) \rightarrow B \ell_{P_{c}}\left(B \ell_{P_{\mu}} P\right) .
$$

Let $E_{3}$ be its exceptional divisor. On geometric fibers over the gerbe of the root stack $E_{3}$ consists of components $Q_{u v}, Q_{v w}, Q_{u w}$, fibered over $\tilde{\lambda}_{u v}$, etc. With an exact sequence of normal bundles, as in the second blowup, we see that each component is isomorphic to $\mathbb{P}^{1} \times \mathbb{P}^{1}$, with $\mathcal{O}\left(E_{3}\right)$ of degree -2 along ruling sections and -1 along fibers, and is glued along a fiber to the exceptional divisor $e_{u}$ of a blow-up of $\widetilde{E}_{u}$, etc., along another fiber to the exceptional divisor $f_{u}$ of a blow-up of $F_{u}$, etc., and along a ruling section (in the table, $\tilde{\lambda}_{v w}$, etc.) to $\Sigma_{3} \cong \Sigma_{2}$.

\begin{tabular}{c|cccccc} 
& $\tilde{d}_{u}$ & $e_{u}$ & $\tilde{\lambda}_{v w}$ & $c_{u}$ & $\tilde{f}_{2, u}$ & $f_{u}$ \\
\hline$\omega^{\vee}$ & 0 & 0 & 3 & 0 & 0 & 0 \\
$E_{1}$ & -1 & 0 & 2 & -1 & 0 & 0 \\
$E_{2}$ & 1 & 0 & 1 & 1 & -1 & 0 \\
$E_{3}$ & 1 & -1 & -2 & 0 & 1 & -1
\end{tabular}

Multiples of $\gamma\left(\beta\left(\alpha \omega^{\vee}-E_{1}\right)-E_{2}\right)-E_{3}$ with

$$
\alpha>\frac{2}{3}+\frac{1}{3 \beta}-\frac{2}{3 \beta \gamma}, \quad \beta>1+\frac{1}{\gamma}, \quad \text { and } \quad \gamma>1
$$

are relatively ample. 
The degree 8 components of geometric fibers over the root stack $\widetilde{E}_{u}$, $\widetilde{E}_{v}, \widetilde{E}_{w}, Q_{u v}, Q_{v w}, Q_{u w}$ comprise the divisor

$$
\widetilde{E}_{1} \cup E_{3}
$$

in the class of $E_{1}-E_{2}+E_{3}$, with evident contraction $\psi$ to a stack $Z$, isomorphic to each of the intersections $\widetilde{E}_{1} \cap E_{2}, \widetilde{E}_{1} \cap E_{3}, E_{2} \cap E_{3}$. Proposition A.9 is applicable to the contraction $\psi: \widetilde{E}_{1} \cup E_{3} \rightarrow Z$ with the relatively ample line bundle associated with (an integral multiple of) $\gamma\left(\beta\left(\alpha \omega^{\vee}-E_{1}\right)-E_{2}\right)-E_{3}$ for

$$
\alpha \beta>1, \quad(1-\alpha) \beta<1, \quad(2 \alpha-1) \beta<1, \quad \gamma=\frac{1}{(1-\alpha) \beta},
$$

and value of $m$, coefficient appearing in the twist of the relatively ample line bundle by $m\left(E_{1}-E_{2}+E_{3}\right)$, equal to (the same integral multiple of) $(\alpha \beta-1) /(1-\alpha) \beta$. We obtain a contraction

$$
\nu: B \ell_{\widetilde{P}_{\lambda}}\left(B \ell_{P_{c}}\left(B \ell_{P_{\mu}} P\right)\right) \rightarrow P_{1},
$$

restricting to $\psi: \widetilde{E}_{1} \cup E_{3} \rightarrow Z \subset P_{1}$, with $\mathcal{I}_{Z} / \mathcal{I}_{Z}^{2}$ locally free of rank 3 .

By Remark A.11, the effective Cartier divisor $E_{1}+E_{3}$, viewed as invertible sheaf with global section, pushes forward under $\nu$ to a Cartier divisor on $P_{1}$ that we denote by $\Psi$. Over $\operatorname{Spec}(B)$, where we have an isomorphism (5.1) and therefore individually defined divisors $\widetilde{E}_{u}$, etc., further Cartier divisors arise by pushing forward

$$
\widehat{G}_{u}:=2 \widetilde{E}_{u}+\widetilde{E}_{v}+3 \widetilde{F}_{u}+2 Q_{u v}+Q_{u w}
$$

and the analogously defined $\widehat{G}_{v}$ and $\widehat{G}_{w}$ to

$$
\widehat{P}_{1}:=\operatorname{Spec}(B) \times_{S} P_{1} \text {. }
$$

So the union of the blown up $\mathbb{F}_{2}$ components of $\widehat{P}_{1}$ over the gerbe of the root stack is a Cartier divisor, as is 3 times each individual blown up $\mathbb{F}_{2}$ component. Since $\nu$ is a birational contraction of a normal scheme, $P_{1}$ is normal.

We claim, $P_{1}$ has $A_{2}$-singularities along $Z$. This is a local assertion, so we may work over $\widehat{P}_{1}$, which is a quotient stack by $\mu_{3}$. We use the language of schemes (with $\mu_{3}$-action) in the following argument. Fix a closed point $\hat{r} \in \widehat{Z}$ over $s^{\prime} \in \operatorname{Spec}\left(B^{\prime}\right)$, corresponding to some $\mathfrak{m}^{\prime} \subset B^{\prime}$, over a given $s \in D$. Let $n=\operatorname{dim}\left(B_{\mathfrak{m}^{\prime}}^{\prime}\right)$, and let $t, g_{1}, \ldots, g_{n-1}$ be a regular system of parameters, i.e., elements that form a basis of $\mathfrak{m}^{\prime} / \mathfrak{m}^{\prime 2}$. If $\hat{\mathfrak{n}}$ denotes the maximal ideal of the local ring $\mathcal{O}_{\widehat{P}_{1}, \hat{r}}$, where the residue field of $\hat{r}$ is a finite extension $\ell$ of the residue field $k$ of $s^{\prime}$, then since $\widehat{Z}$ is 
regular there is the exact sequence of vector spaces over $\ell[29,16.9 .13]$

$$
0 \rightarrow \mathcal{I}_{\widehat{Z}} / \hat{\mathfrak{n}} \mathcal{I}_{\widehat{Z}} \rightarrow \hat{\mathfrak{n}} / \hat{\mathfrak{n}}^{2} \rightarrow \hat{\mathfrak{n}} /\left(\mathcal{I}_{\widehat{Z}}+\hat{\mathfrak{n}}^{2}\right) \rightarrow 0
$$

from which we learn

$$
\operatorname{dim}\left(\hat{\mathfrak{n}} / \hat{\mathfrak{n}}^{2}\right)=n+3
$$

As well, by Proposition A.9, we have the compatibility of the contraction $\hat{\nu}$ with base change, e.g., via $\left\{s^{\prime}\right\} \rightarrow \operatorname{Spec}\left(B^{\prime}\right)$, and this way we learn that the fiber of $\widehat{P}_{1}$ over $s^{\prime}$ consists of a singular cubic surface of type $3 \mathrm{~A}_{2}$ joined to three components, each isomorphic to the blow-up of $\mathbb{F}_{2}$ at one point. In particular,

$$
\operatorname{dim}\left(\hat{\mathfrak{n}} /\left(\mathfrak{m}^{\prime} \mathcal{O}_{\widehat{P}_{1}, \hat{r}}+\hat{\mathfrak{n}}^{2}\right)\right)= \begin{cases}4, & \text { if } 3 \text { components meet at } \hat{r} \\ 3, & \text { otherwise }\end{cases}
$$

In both cases, we let $x$ and $y$ be local defining equations of the Cartier divisors on $\widehat{P}_{1}$, each consisting (on fibers) of one blown up $\mathbb{F}_{2}$ component with multiplicity 3 , and $z$, of their union with multiplicity 1 ; in the case 3 components meet at $\hat{r}$ we additionally let $q$ be a local defining equation for the singular cubic surface divisor component.

We have, after adjusting by units,

$$
x y=z^{3}
$$

and, in the two cases,

$$
t=q z \in \hat{\mathfrak{n}}^{2}, \quad \text { respectively, } \quad t=z .
$$

The kernel

$$
\left(\mathfrak{m}^{\prime} \mathcal{O}_{\widehat{P}_{1}, \hat{r}}+\hat{\mathfrak{n}}^{2}\right) / \hat{\mathfrak{n}}^{2}
$$

of the canonical homomorphism $\hat{\mathfrak{n}} / \hat{\mathfrak{n}}^{2} \rightarrow \hat{\mathfrak{n}} /\left(\mathfrak{m}^{\prime} \mathcal{O}_{\widehat{P}_{1}, \hat{r}}+\hat{\mathfrak{n}}^{2}\right)$ is generated by $\mathfrak{m}^{\prime}$. So the kernel is, in the two cases, of dimension $n-1$ with basis $g_{1}, \ldots, g_{n-1}$, respectively, of dimension $n$ with basis $t, g_{1}, \ldots, g_{n-1}$.

Suppose, first, $\hat{r}$ is the point where three components come together. We know, modulo $q$, the point $\hat{r}$ is an $\mathrm{A}_{2}$-singular point of the cubic surface component, with $\hat{\mathfrak{n}} /\left(\mathfrak{m}^{\prime} \mathcal{O}_{\widehat{P}_{1}, \hat{r}}+q \mathcal{O}_{\widehat{P}_{1}, \hat{r}}+\hat{\mathfrak{n}}^{2}\right)$ of dimension 3 , spanned by $x, y$, and $z$. So $q, x, y$, and $z \operatorname{span} \hat{\mathfrak{n}} /\left(\mathfrak{m}^{\prime} \mathcal{O}_{\widehat{P}_{1}, \hat{r}}+\hat{\mathfrak{n}}^{2}\right)$; they define a morphism to $\mathbb{A}_{B^{\prime}}^{4}$ which is unramified at $\hat{r}$ and hence by $[29,18.4 .7]$ restricts and lifts to a closed immersion of a neighborhood $\operatorname{Spec}(C)$ of $\hat{r}$ in an étale affine neighborhood $\operatorname{Spec}(\Delta)$ of the point 0 over $s^{\prime}$ in $\mathbb{A}_{B^{\prime}}^{4}$; let $\delta_{q}, \delta_{x}, \delta_{y}, \delta_{z} \in \Delta$ denote the coordinate functions from $\mathbb{A}_{B^{\prime}}^{4}$. Then, setting $\widetilde{\Delta}:=\Delta /\left(t-\delta_{q} \delta_{z}\right)$, we see that $\Delta \rightarrow C$ factors through

$$
\widetilde{\Delta} /\left(\delta_{x} \delta_{y}-\delta_{z}^{3}\right)
$$


The element $\delta_{x} \delta_{y}-\delta_{z}^{3}$ is irreducible in the local ring of $\widetilde{\Delta}$ at the point 0 over $s^{\prime}$, so the localization of (5.3) is an integral domain, and we have found the desired local form of the singularity at $\hat{r}$.

Now suppose $\hat{r}$ is a point of the component $\mathbb{P}^{1}$ where two blown up $\mathbb{F}_{2}$ components meet. The Cartier divisor defined by $x$, restricted to the other blown up $\mathbb{F}_{2}$ component, defines $\mathbb{P}^{1}$ with multiplicity 1 , and the same is true with $y$ and the first blown up $\mathbb{F}_{2}$ component. It follows that $x, y$, and a polynomial of degree $[\ell: k]$ in a local parameter $p$ for $\mathbb{P}^{1}$ span $\hat{\mathfrak{n}} /\left(\mathfrak{m}^{\prime} \mathcal{O}_{\widehat{P}_{1}, \hat{r}}+\hat{\mathfrak{n}}^{2}\right)$. We argue as above, with morphism to $\mathbb{A}_{B^{\prime}}^{3}$ determined by $x, y$, and $p$ and $\Delta \rightarrow C$ factoring through $\Delta /\left(\delta_{x} \delta_{y}-t^{3}\right)$.

On $P_{1}$ we have Cartier divisors $\Psi \cup \nu\left(\Sigma_{3}\right)$ and $\Psi$. Hence $\nu\left(\Sigma_{3}\right)$ is a Cartier divisor, which becomes linearly equivalent to $-\Psi$ after base change to any affine étale chart of $\sqrt[3]{(S, D)}$ where the pre-image of the gerbe of the root stack is principal. We apply Proposition A.9 with relatively ample $\mathcal{O}_{P_{1}}(1)$ and $m$ taken so that the twist by $m \nu\left(\Sigma_{3}\right)$ has degree 0 on $\tilde{f}_{2, u}$. Thus we obtain a contraction

$$
\rho: P_{1} \rightarrow P_{2},
$$

where $P_{2}$ is a Brauer-Severi surface bundle over $\sqrt[3]{(S, D)}$. Under $\rho$ we have $\nu\left(\Sigma_{3}\right)$ contracting to a copy $G$ in $P_{2}$ of the gerbe of the root stack, with $\mathcal{I}_{G} / \mathcal{I}_{G}^{2}$ locally free of rank 4 . An exact sequence analogous to (5.2) shows that $\operatorname{dim}\left(\hat{\mathfrak{n}} / \hat{\mathfrak{n}}^{2}\right)=n+3$, where $\hat{\mathfrak{n}}$ is the maximal ideal of the local ring, after base change to an affine étale chart of $\sqrt[3]{(S, D)}$, at a closed point $\hat{r}$ over $G$; in particular, $P_{2}$ has hypersurface singularities.

As above, we may work over $\operatorname{Spec}(B)$ and perform previous analysis to show that 3 times each $\mathbb{F}_{1}$ component over the gerbe of the root stack is a Cartier divisor. This leads to an étale local defining equation at a point $\hat{r}$ as above of $\delta_{x} \delta_{y} \delta_{z}-t^{3}$, with analogous notation to that appearing in (5.3). With notation as before, we have an affine chart $\operatorname{Spec}(C)$ of

$$
\widehat{P}_{2}:=\operatorname{Spec}(B) \times_{S} P_{2},
$$

which we may without loss of generality take to be $\mu_{3}$-invariant, and a closed immersion

$$
\operatorname{Spec}(C) \rightarrow \operatorname{Spec}(\Delta)
$$

identifying the local ring $C_{\hat{\mathfrak{n}}}$ with $\Delta_{\mathfrak{\mathfrak { n }}^{\prime}} /\left(\delta_{x} \delta_{y} \delta_{z}-t^{3}\right)$, where $\hat{\mathfrak{n}}^{\prime}$ denotes the maximal ideal, corresponding to the image of $\hat{r}$ in $\operatorname{Spec}(\Delta)$. Although $\Delta$ is not determined canonically, its strict henselization at $\hat{\mathfrak{n}}^{\prime}$ is canonically determined (up to fixing a separable closure of the residue field): it is a strict henselization of affine 3-space over $B^{\prime}$.

By Proposition 2.5, there exists a scheme $P_{3}$, Fano over $S$, with

$$
\sqrt[3]{(S, D)} \times{ }_{S} P_{3} \cong P_{2}
$$


By Remark 2.6, $P_{2} \rightarrow P_{3}$ is a coarse moduli space, so with the above notation, $P_{3}$ has affine coordinate ring $C^{\mu_{3}}$. We claim, $P_{3}$ is regular; equivalently,

$$
\widehat{P}_{3}:=\operatorname{Spec}(B) \times_{S} P_{3}
$$

is regular. It suffices to verify the claim after passing to a strict henselization $\left(C^{\mu_{3}}\right)^{s h}$ of $P_{3}$. By [29, 18.8.10], we have

$$
C \otimes_{C^{\mu_{3}}}\left(C^{\mu_{3}}\right)^{s h} \cong C^{s h}
$$

Then a strict Henselization of $P_{2}$ (for Henselization of a stack, see [4, $\S 2.7])$ takes the form

$$
\left[\operatorname{Spec}(C) / \mu_{3}\right] \times_{\operatorname{Spec}\left(C^{\mu_{3}}\right)} \operatorname{Spec}\left(\left(C^{\mu_{3}}\right)^{s h}\right) \cong\left[\operatorname{Spec}\left(C^{s h}\right) / \mu_{3}\right] .
$$

We have, $\mu_{3}$-equivariantly,

$$
C^{s h} \cong \Delta^{s h} /\left(\delta_{x} \delta_{y} \delta_{z}-t^{3}\right)
$$

at a point where three components meet. Hence upon taking $\mu_{3}$-invariants,

$$
\left(C^{\mu_{3}}\right)^{s h} \cong\left(C^{s h}\right)^{\mu_{3}} \cong\left(\Delta^{s h}\right)^{\mu_{3}} /\left(\delta_{x} \delta_{y} \delta_{z}-f\right) .
$$

The ring $\left(\Delta^{s h}\right)^{\mu_{3}}$ is a strict Henselization of affine 3-space over $B$ and hence is regular. A similar analysis takes care of the regularity at a point where two components meet. So, $P_{3}$ is regular.

\section{REVERSE CONSTRUCTION}

In this section, we start with a mildly degenerating Brauer-Severi surface bundle and carry out the reverse construction of Proposition 4.4.

For every $s \in D$, the fiber $X_{s}$ has, geometrically, three irreducible components. Lemma 2.9 determines a degree 3 finite étale cover

$$
\widetilde{D}:=\pi_{0}\left(X^{\mathrm{sm}} / S\right) \times_{S} D \rightarrow D .
$$

For $s \in D$, let $\operatorname{Spec}(B) \rightarrow S$ be an étale neighborhood of $s$ that trivializes $\widetilde{D} \rightarrow D$. By Remark 2.10, the three sections of

$$
\operatorname{Spec}(B) \times_{S} \widetilde{D} \rightarrow \operatorname{Spec}(B) \times_{S} D
$$

determine three divisors $W_{1}, W_{2}, W_{3}$ on $\operatorname{Spec}(B) \times_{S} X$, whose intersections with the fiber $X_{s}$ of any $s \in \operatorname{Spec}(B) \times_{S} D$ are the irreducible components of $X_{s}$. Let $w_{1}, w_{2}, w_{3}$ be respective local defining equations of the divisors near a singular point $r \in X_{s}$. Then, after modification by a unit, we have $f=w_{1} w_{2} w_{3}$.

We claim, there is an étale local equation

$$
w_{1} w_{2} w_{3}-f, \quad \text { respectively, } \quad w_{1} w_{2}-f,
$$

(after suitable renumbering of indices) for $\operatorname{Spec}(B) \times{ }_{S} X$ in $\mathbb{A}_{B}^{3}$, according to whether $x$ is a point of $X_{s}$ where 3 or 2 components meet. 
To establish the claim, we argue as in Section 5. Let $\mathfrak{m} \subset B$ be the maximal ideal corresponding to $s$ and $n=\operatorname{dim}\left(B_{\mathfrak{m}}\right)$. We may choose a regular system of parameters for $B_{\mathfrak{m}}$ of the form $f, g_{1}, \ldots, g_{n-1}$. The space $\mathfrak{n} / \mathfrak{n}^{2}$ has dimension $n+2$. Since the fiber $X_{s}$ has hypersurface singularities, at a singular point $r \in X_{s}$ with corresponding maximal ideal $\mathfrak{n} \subset \mathcal{O}_{X, r}$ we have

$$
\operatorname{dim}\left(\mathfrak{n} /\left(\mathfrak{m} \mathcal{O}_{X, r}+\mathfrak{n}^{2}\right)\right)=3
$$

The claim follows, as in Section 5, once we show that $\mathfrak{n} /\left(\mathfrak{m} \mathcal{O}_{X, r}+\mathfrak{n}^{2}\right)$ is spanned by $w_{1}, w_{2}, w_{3}$, respectively by two of them and a local parameter for the component $\mathbb{P}^{1}$ of the singular locus of the fiber.

We have

$$
\operatorname{dim}\left(\left(\mathfrak{m} \mathcal{O}_{X, r}+\mathfrak{n}^{2}\right) / \mathfrak{n}^{2}\right)=n-1
$$

The image of $f$ in $\mathcal{O}_{X, r}$ lies in $\mathfrak{n}^{2}$. Indeed, if not, then $\mathcal{O}_{X, r} /(f)$ would be regular, hence also the further localization, where we pass to the residue field at the generic point of $D$, would be regular. But the relative singular locus of $X \times_{S} D$ over $D$ is flat (since it has constant Hilbert polynomial), hence some point of the singular locus over the generic point of $D$ specializes to $r$, contradiction. So, the images of $g_{1}, \ldots, g_{n-1}$ form a basis of $\left(\mathfrak{m} \mathcal{O}_{X, r}+\mathfrak{n}^{2}\right) / \mathfrak{n}^{2}$. Now, modulo $g_{1}, \ldots, g_{n-1}$, we still have $f=w_{1} w_{2} w_{3}$, where $f=0$ now defines the fiber $X_{s}$, and hence $w_{1}, w_{2}$, $w_{3}$ define its three irreducible components, each with multiplicity one.

Suppose, first, that $r$ is the point where all three components meet. Then, for all $\{i, j, k\}=\{1,2,3\}$ the classes of $w_{j}$ and $w_{k}$ are a basis of $\mathfrak{n} /\left(\mathfrak{m} \mathcal{O}_{X, r}+w_{i} \mathcal{O}_{X, r}+\mathfrak{n}^{2}\right)$. So, the classes of $w_{1}, w_{2}, w_{3}$ are a basis of $\mathfrak{n} /\left(\mathfrak{m} \mathcal{O}_{X, r}+\mathfrak{n}^{2}\right)$, and we are done in this case.

If $r$ is a point where two components meet, say, those defined by $w_{1}$ and $w_{2}$, then for $\{i, j\}=\{1,2\}$ and local parameter $p$ for the component $\mathbb{P}^{1}$ of the singular locus, $w_{j}$ and $p$ are a basis of $\mathfrak{n} /\left(\mathfrak{m} \mathcal{O}_{X, r}+w_{i} \mathcal{O}_{X, r}+\mathfrak{n}^{2}\right)$. It follows that $w_{1}, w_{2}, p$ span $\mathfrak{n} /\left(\mathfrak{m} \mathcal{O}_{X, r}+\mathfrak{n}^{2}\right)$, and we are done in this case as well.

The claim implies the corresponding singularity type of $\sqrt[3]{(S, D)} \times{ }_{S} X$, where we have $f=t^{3}$ in the coordinate ring of an affine étale chart. The two blowing up steps therefore supply a resolution of singularities of $\sqrt[3]{(S, D)} \times_{S} X$. The three contractions then may be performed, as in Section 5, noticing by the normal cone description of Proposition A.9 that the outcome of each contraction is regular. 


\section{Regular DEGENERACY LOCUS IMPLIES MILD DEGENERATION}

In this section we complete the proof of Proposition 4.4 by showing that a Brauer-Severi surface bundle $\pi: X \rightarrow S$ with $X$ regular and singular fibers precisely along a regular divisor $D \subset S$, always has mild degeneration. We may assume that $S$ is quasi-compact and integral. We consider various cases, depending on the dimension of $S$.

If $\operatorname{dim}(S)=1$ then $\operatorname{dim}(X)=3$, hence if $s \in S$ is any closed point then 3 is an upper bound on the embedding dimension of $X_{s}$ at any of its closed points. So $X \rightarrow S$ has mild degeneration.

If $\operatorname{dim}(S)=2$ then by the previous case we know that the fiber at the generic point of every component of $D$ is, geometrically, a union of three Hirzebruch surfaces. So, there is an open subscheme $S^{\prime}$ of $S$ such that $S^{\prime} \times{ }_{S} X \rightarrow S^{\prime}$ has mild degeneration and $\operatorname{dim}\left(S \backslash S^{\prime}\right)=0$.

We apply the reverse construction (Section 6) to $S^{\prime} \times_{S} X \rightarrow S^{\prime}$ to obtain a smooth $\mathbb{P}^{2}$-bundle

$$
P^{\prime} \rightarrow S^{\prime} \times_{S} \sqrt[3]{(S, D)}
$$

By Proposition 2.1 (iii), this extends to a $\mathbb{P}^{2}$-bundle

$$
P \rightarrow \sqrt[3]{(S, D)}
$$

We now apply the forwards construction to obtain a mildly degenerating Brauer-Severi surface bundle $\hat{\pi}: \widehat{X} \rightarrow S$.

By Remark 4.2 and the fact that a line bundle, ample on fibers, is relatively ample, we have the ampleness of the dual of the relative dualizing sheaf of $\widehat{X} \rightarrow S$. This holds as well for $X \rightarrow S$, where the ampleness on geometric fibers over $S \backslash S^{\prime}$ is dealt with by [27, 4.5.14], which tells us that ampleness of a line bundle on a Noetherian scheme is independent of any non-reduced structure, in combination with the fact that a cone over a twisted cubic curve has Picard group isomorphic to $\mathbb{Z}$.

Since

$$
X \cong \operatorname{Proj}\left(\bigoplus_{n \geq 0} \pi_{*}\left(\omega_{X / S}^{\vee}\right)^{n}\right) \quad \text { and } \quad \widehat{X} \cong \operatorname{Proj}\left(\bigoplus_{n \geq 0} \hat{\pi}_{*}\left(\omega_{\widehat{X} / S}^{\vee}\right)^{n}\right),
$$

where the direct image sheaves are locally free for all $n \gg 0$ with isomorphic restrictions over $S^{\prime}$, they are isomorphic for all $n \gg 0$, hence $X \cong \widehat{X}$ is a Brauer-Severi surface bundle with mild degeneration.

We observe that the argument for $\operatorname{dim}(S)=2$ does not use the regularity of $X$, but only the flatness of $X \rightarrow S$ and the regularity of $S^{\prime} \times{ }_{S} X$.

The case $\operatorname{dim}(S) \geq 3$ reduces to the case $\operatorname{dim}(S)=2$ by slicing the base. Let $s \in S$ be a point where the geometric fiber is irreducible with reduced subscheme a cone over a twisted cubic curve. Let $f, g_{1}, \ldots$, $g_{d-1}$ be a regular system of parameters for $\mathcal{O}_{S, s}$, and let $T$ be the regular 
two-dimensional scheme $\operatorname{Spec}\left(\mathcal{O}_{S, s} /\left(g_{2}, \ldots, g_{d-1}\right)\right)$. Let $Y:=T \times_{S} X$. We claim, $Y \backslash Y_{s}$ is regular. This is clear at points in the relative smooth locus of $Y \backslash Y_{s} \rightarrow T \backslash\{s\}$. Consider the generic point $\eta$ of $T \cap D$ with corresponding maximal ideal $\mathfrak{m} \subset \mathcal{O}_{S, \eta}$, and a closed point $r \in\left(X_{\eta}\right)^{\text {sing }}$, with corresponding maximal ideal $\mathfrak{n} \subset \mathcal{O}_{X, r}$. We use the exact sequence

$$
0 \rightarrow\left(\mathfrak{m} \mathcal{O}_{X, r}+\mathfrak{n}^{2}\right) / \mathfrak{n}^{2} \rightarrow \mathfrak{n} / \mathfrak{n}^{2} \rightarrow \mathfrak{n} /\left(\mathfrak{m} \mathcal{O}_{X, r}+\mathfrak{n}^{2}\right) \rightarrow 0 .
$$

The space in the middle has dimension $d+1$, on the right, 3 , hence on the left, $d-2$. The space $\mathfrak{m} / \mathfrak{m}^{2}$ has dimension $d-1$ and is spanned by $f, g_{2}, \ldots, g_{d-1}$. Moreover, $f$ lies in the kernel of

$$
\mathfrak{m} / \mathfrak{m}^{2} \rightarrow\left(\mathfrak{m} \mathcal{O}_{X, r}+\mathfrak{n}^{2}\right) / \mathfrak{n}^{2} .
$$

So $g_{2}, \ldots, g_{d-1}$ are linearly independent in $\mathfrak{n} / \mathfrak{n}^{2}$, and hence $\mathcal{O}_{Y, r}$ is regular.

By the observation made after the argument for the case $\operatorname{dim}(S)=$ 2 , we may conclude that $Y \rightarrow T$ has mild degeneration, and this is a contradiction.

\section{LOCAL ANALYSIS I}

For the next result we use the notation of Section 5 but work with schemes with $\mu_{3}$-action: $P^{\prime}$ will denote $\operatorname{Proj}\left(B^{\prime}[u, v, w]\right)$, so $\widehat{P}=\left[P^{\prime} / \mu_{3}\right]$, etc.

Proposition 8.1. With the notation as remarked above, $\omega_{P_{2}^{\prime} / \operatorname{Spec}\left(B^{\prime}\right)}^{\vee}$ is very ample over $\operatorname{Spec}\left(B^{\prime}\right)$, and formation of its global sections commutes with base change to an arbitrary Noetherian scheme over $\operatorname{Spec}\left(B^{\prime}\right)$. Identifying the pullback of $\omega_{P_{2}^{\prime} / \operatorname{Spec}\left(B^{\prime}\right)}^{\vee}$ to $B \ell_{\widetilde{P}_{\lambda}^{\prime}}\left(B \ell_{P_{c}^{\prime}}\left(B \ell_{P_{\mu}^{\prime}} P^{\prime}\right)\right)$ with

$$
\omega^{\vee}-2 E_{1}-E_{2}-E_{3}
$$

and sections with cubic forms in $u, v, w$ vanishing appropriately along the $E_{i}$, the sections form a free $B^{\prime}$-module with basis $t^{3} w^{3}, t^{2} u w^{2}, \quad t v w^{2}, \quad t u^{2} w, \quad u v w, t^{2} v^{2} w, t^{3} u^{3}, t^{2} u^{2} v, \quad t u v^{2}, t^{3} v^{3}$.

The identification of line bundles in Proposition 8.1 comes from the observation that the line bundle associated with $\omega^{\vee}-2 E_{1}-E_{2}-E_{3}$ is the dual of the relative dualizing sheaf of $B \ell_{\widetilde{P}_{\lambda}^{\prime}}\left(B \ell_{P_{c}^{\prime}}\left(B \ell_{P_{\mu}^{\prime}} P^{\prime}\right)\right)$ over $\operatorname{Spec}\left(B^{\prime}\right)$ (by standard behavior of dualizing sheaf under blowing up) and under the morphism to $P_{2}^{\prime}$ has direct image $\omega_{P_{2}^{\prime} / \operatorname{Spec}\left(B^{\prime}\right)}^{\vee}$.

Proof. The observation from Remark 4.2, that the line bundle $\omega_{P_{2}^{\prime} / \operatorname{Spec}\left(B^{\prime}\right)}^{\vee}$ on fibers is very ample with space of global sections of dimension 10, implies the assertions in the first statement. 
For the remaining assertion, we compute with local charts. We work, first, over the open subscheme

$$
W^{\prime}:=\operatorname{Spec}\left(B^{\prime}[u, v]\right)
$$

of $P^{\prime}$ and use notation with the letter $P$ replaced by $W$ to denote restriction over $W^{\prime}$. The blowup $B \ell_{W_{\mu}^{\prime}} W^{\prime}$ is covered by three charts:

Chart 1: coordinates $u, t_{1}, v_{1}$ with $t=u t_{1}$ and $v=u v_{1}$. Equation for $E_{1}: u=0$; equation for $W_{c}^{\prime}: u=v_{1}=0$.

Chart 2: coordinates $v, t_{2}, u_{2}$ with $t=v t_{2}$ and $u=v u_{2}$. Equation for $E_{1}: v=0$; trivial intersection with $W_{c}^{\prime}$, equation for $\widetilde{W}_{\lambda}^{\prime}: t_{2}=u_{2}=0$.

Chart 3: coordinates $t, u_{3}, v_{3}$ with $u=t u_{3}$ and $v=t v_{3}$. Equation for $E_{1}: t=0$; equation for $W_{c}^{\prime}: t=v_{3}=0$.

The next blowup $B \ell_{W_{c}^{\prime}}\left(B \ell_{W_{\mu}^{\prime}} W^{\prime}\right)$ is covered by five charts, Chart 2 and:

Chart 1': coordinates $u, t_{1}, v_{1}^{\prime}$ with $v_{1}=u v_{1}^{\prime}$. Equation for $E_{2}: u=0$; equation for $\widetilde{W}_{\lambda}^{\prime}: t_{1}=v_{1}^{\prime}=0$.

Chart 1": coordinates $t_{1}, v_{1}, u^{\prime \prime}$ with $u=v_{1} u^{\prime \prime}$. Equation for $E_{2}$ : $v_{1}=0$; trivial intersection with $\widetilde{W}_{\lambda}^{\prime}$.

Chart $3^{\prime}$ : (analogous)

Chart $3^{\prime \prime}$ : (analogous)

The next blowup $B \ell_{\widetilde{W}_{\lambda}^{\prime}}\left(B \ell_{W_{c}^{\prime}}\left(B \ell_{W_{\mu}^{\prime}} W^{\prime}\right)\right)$ is covered by seven charts, of which we need just two:

Chart $1_{\mathrm{a}}^{\prime}$ : coordinates $u, t_{1}, v_{1, \mathrm{a}}^{\prime}$ with $v_{1}^{\prime}=t_{1} v_{1, \mathrm{a}}^{\prime}$. Equation for $E_{3}$ : $t_{1}=0$.

Chart $2_{\mathrm{a}}$ : coordinates $v, t_{2}, u_{2, \mathrm{a}}$ with $u_{2}=t_{2} u_{2, \mathrm{a}}$. Equation for $E_{3}$ : $t_{2}=0$

The union of charts $1_{\mathrm{a}}^{\prime}$ and $2_{\mathrm{a}}$ contains the generic point of every component of $E_{1}, E_{2}$, and $E_{3}$ over $W^{\prime}$. With cyclic shifts of variables $u, v, w$ we have additional affine opens $U^{\prime}$ and $V^{\prime}$ of $P^{\prime}$ with analogous charts of the blow-ups. By considering the analogous charts, too, we find that sections of $\omega^{\vee}-2 E_{1}-E_{2}-E_{3}$ are cubic forms in $u, v, w$ with coefficients in $B^{\prime}$, such that after applying any cyclic permutation of $u$, $v, w$ and setting $w$ to 1 the image in the coordinate ring of Chart $1_{\mathrm{a}}^{\prime}$ lies in the ideal

$$
u^{3} t_{1} B\left[u, t_{1}, v_{1, \mathrm{a}}^{\prime}\right] /\left(u^{3} t_{1}^{3}-f\right),
$$

and the image in the coordinate ring of Chart $2_{\mathrm{a}}$ lies in the ideal

$$
v^{2} t_{2} B\left[v, t_{2}, u_{2, \mathrm{a}}\right] /\left(v^{3} t_{2}^{3}-f\right) \text {. }
$$


We compute with respect to the basis of degree 3 monomials in $u, v$, $w$. The kernel of

$$
B^{\prime 10} \rightarrow(B / f)\left[u, t_{1}, v_{1, \mathrm{a}}^{\prime}\right] /\left(u^{3} t_{1}\right)
$$

may be computed with the help of the observation that the map factors through $\left((B / f)[t] /\left(t^{3}\right)\right)^{10}$, which along with $(B / f)\left[u, t_{1}, v_{1, \mathrm{a}}^{\prime}\right] /\left(u^{3} t_{1}\right)$ is a free $B / f$-module. The kernel of

$$
B^{\prime 10} \rightarrow(B / f)\left[v, t_{2}, u_{2, \mathrm{a}}\right] /\left(v^{2} t_{2}\right)
$$

may be computed similarly. These computations lead to the determination of the module of such forms as the free module with the claimed basis.

\section{LOCAL ANALYSIS II}

We exhibit a Brauer-Severi surface fibration over a two-dimensional base, with singular fibers along a union of two intersecting divisors and smooth total space. For conic bundles this is easy:

$$
\operatorname{Proj}\left(k[s, t, x, y, z] /\left(s x^{2}+t y^{2}+z^{2}\right)\right) .
$$

This conic bundle over $\mathbb{A}^{2}=\operatorname{Spec}(k[s, t])$ has smooth total space and singular fibers along the divisor defined by $s t=0$.

For Brauer-Severi surface bundles this is more complicated. Over the generic point, the fiber is an anticanonically embedded Brauer-Severi surface, defined in $\mathbb{P}^{9}$ by

$$
\frac{10 \cdot 11}{2}-h^{0}\left(\mathbb{P}^{2},\left(\omega_{\mathbb{P}^{2}}^{\vee}\right)^{2}\right)=55-28=27
$$

quadratic equations. We will obtain a equations for a Brauer-Severi surface bundle over $\mathbb{A}^{2}$ with smooth total space and singular fibers along the union of coordinate axes by applying results from $\S 4$. The existence of such a Brauer-Severi surface bundle has been shown by Maeda [47] using different methods (ideals and orders in a central simple algebra).

Let $k$ be a field of characteristic different from 3 containing a primitive cube root of unity $\zeta$, and let $\mu_{3} \times \mu_{3}$ act on $\mathbb{A}^{2} \times \mathbb{P}^{2}$ over $k$, where cube root of unity $\zeta$ in the first factor acts by

$$
(s, t, u: v: w) \mapsto\left(\zeta s, t, \zeta u: \zeta^{2} v: w\right)
$$

and in the second factor acts by

$$
(s, t, u: v: w) \mapsto(s, \zeta t, w: u: v) .
$$

Then

$$
\left[\left(\mathbb{A}^{2} \backslash\{0\}\right) \times \mathbb{P}^{2} / \mu_{3} \times \mu_{3}\right]
$$


is a smooth $\mathbb{P}^{2}$-fibration satisfying the hypotheses of Proposition 4.4. There is a corresponding mildly degenerating Brauer-Severi surface bundle

$$
\pi: X \rightarrow \mathbb{A}^{2} \backslash\{0\}
$$

By Proposition 8.1 applied to the pullback

$$
\pi^{\prime}: X^{\prime} \rightarrow \mathbb{A}^{2} \backslash\{0\}
$$

of $\pi$ by $(s, t) \mapsto\left(s^{3}, t^{3}\right)$, first restricted over $\mathbb{A}^{1} \times\left(\mathbb{A}^{1} \backslash\{0\}\right)$ and then restricted $\left(\mathbb{A}^{1} \backslash\{0\}\right) \times \mathbb{A}^{1}$, the space of global sections of $\omega_{X^{\prime} / \mathbb{A}^{2} \backslash\{0\}}^{\vee}$ is the intersection of

$\mathcal{S}:=$

$$
k\left[s, t, t^{-1}\right]\left\langle s^{3} w^{3}, s^{2} u w^{2}, s v w^{2}, s u^{2} w, u v w, s^{2} v^{2} w, s^{3} u^{3}, s^{2} u^{2} v, s u v^{2}, s^{3} v^{3}\right\rangle
$$

and

$$
\begin{aligned}
& \mathcal{T}:= \\
& \quad k\left[s, s^{-1}, t\right]\left\langle t^{3} \tilde{w}^{3}, t^{2} \tilde{u} \tilde{w}^{2}, t \tilde{v} \tilde{w}^{2}, t \tilde{u}^{2} \tilde{w}, \tilde{u} \tilde{v} \tilde{w}, t^{2} \tilde{v}^{2} \tilde{w}, t^{3} \tilde{u}^{3}, t^{2} \tilde{u}^{2} \tilde{v}, t \tilde{u} \tilde{v}^{2}, t^{3} \tilde{v}^{3}\right\rangle,
\end{aligned}
$$

where

$$
\begin{aligned}
\tilde{u} & =\zeta u+\zeta^{2} v+w, \\
\tilde{v} & =\zeta^{2} u+\zeta v+w, \\
\tilde{w} & =u+v+w .
\end{aligned}
$$

Indeed, the action of cyclically permuting the coordinates $u, v, w$ is compatible with the diagonal action by $\zeta, \zeta^{2}, 1$ on $\tilde{u}, \tilde{v}, \tilde{w}$.

Only nonnegative powers of $s$ and $t$ show up in an element of $\mathcal{S} \cap \mathcal{T}$. For a polynomial $f \in k[s, t, u, v, w]$, homogeneous of degree 3 in $u, v, w$ :

- if $s^{3} \mid f$, then $f \in \mathcal{S}$;

- if $t^{3} \mid f$, then $f \in \mathcal{T}$;

- generally, if we write

$$
f=\sum_{a, b \geq 0} s^{a} t^{b} f_{a b}, \quad \text { with } \quad f_{a b} \in k[u, v, w],
$$

for all $a$ and $b$, then $f \in \mathcal{S}$ if and only if $s^{a} f_{a b} \in \mathcal{S}$ for all $a$ and $b$, and $f \in \mathcal{T}$ if and only if $t^{b} f_{a b} \in \mathcal{T}$ for all $a$ and $b$.

We easily characterize the homogeneous $f \in k[u, v, w]$ of degree 3 with $s^{a} f \in \mathcal{S}$ for $a \leq 2$ :

\begin{tabular}{c|cc}
$a$ & $k$-basis \\
\hline 0 & $u v w$ & \\
1 & above and & $u^{2} w, \quad v w^{2}, \quad u v^{2}$ \\
2 & above and & $u w^{2}, \quad v^{2} w, \quad u^{2} v$
\end{tabular}


Similarly, with a bit of linear algebra we characterize the homogeneous $f \in k[u, v, w]$ of degree 3 with $t^{b} f \in \mathcal{T}$ for $b \leq 2$ :

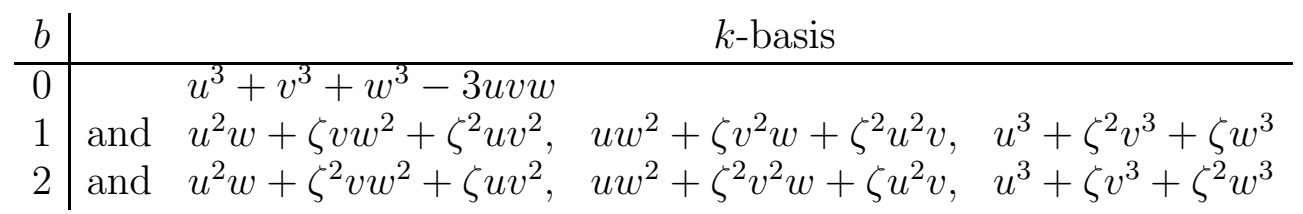

With these data, we determine the space of $f$ with $s^{a} t^{b} f \in \mathcal{S} \cap \mathcal{T}$ for $0 \leq a, b \leq 2$ as trivial when $\min (a, b)=0$ and of dimension $a+b-1$ when $1 \leq a, b \leq 2$. E.g., for $a=b=1$ the only linear combination of $u v w, u^{2} w, v w^{2}, u v^{2}$ that belongs to the space inducated for $b=1$ is, up to scalar multiplication, $u^{2} w+\zeta v w^{2}+\zeta^{2} u v^{2}$. We deduce that $\mathcal{S} \cap \mathcal{T}$ is the free $k[s, t]$-module with basis

$$
\begin{gathered}
t^{3} u v w, \quad s t\left(u^{2} w+\zeta v w^{2}+\zeta^{2} u v^{2}\right), \quad s t^{2}\left(u^{2} w+\zeta^{2} v w^{2}+\zeta u v^{2}\right), \\
s t^{3}\left(u^{2} w+v w^{2}+u v^{2}\right), \quad s^{2} t\left(u w^{2}+\zeta v^{2} w+\zeta^{2} u^{2} v\right), \\
s^{2} t^{2}\left(u w^{2}+\zeta^{2} v^{2} w+\zeta u^{2} v\right), \quad s^{2} t^{3}\left(u w^{2}+v^{2} w+u^{2} v\right), \\
s^{3}\left(u^{3}+v^{3}+w^{3}-3 u v w\right), \quad s^{3} t\left(u^{3}+\zeta^{2} v^{3}+\zeta w^{3}\right), \quad s^{3} t^{2}\left(u^{3}+\zeta v^{3}+\zeta^{2} w^{3}\right) .
\end{gathered}
$$

By Proposition 8.1, the basis of $\mathcal{S} \cap \mathcal{T}$ defines a morphism $X^{\prime} \rightarrow \mathbb{A}^{2} \times \mathbb{P}^{9}$. With linear algebra, we find $f_{1}, \ldots, f_{27} \in k\left[s, t, x_{0}, \ldots, x_{9}\right]$ that are

- homogeneous of degree 2 in $x_{0}, \ldots, x_{9}$ and at most linear in $s, t$.

- zero upon substituting $s^{3}$ for $s, t^{3}$ for $t$, and the $i$ th basis element for $x_{i-1}$, for every $i$,

- linearly independent over $k(s, t)$.

The polynomials are displayed in Table 1 . The conditions imply that

$$
\operatorname{Proj}\left(k(s, t)\left[x_{0}, \ldots, x_{9}\right] /\left(f_{1}, \ldots, f_{27}\right)\right)
$$

is the Brauer-Severi surface that appears as generic fiber of $\pi$.

Lemma 9.1. Let $f_{1}, \ldots, f_{27}$ be as in Table 1, where $k$ is a field of characteristic different from 3 with primitive cube root of unity $\zeta$. Then $k\left[s, t, x_{0}, \ldots, x_{9}\right] /\left(f_{1}, \ldots, f_{27}\right)$ is finitely generated and free as a module over $k\left[s, t, x_{0}, x_{1}, x_{7}\right]$, with basis $1, x_{2}, x_{3}, x_{4}, x_{5}, x_{5}^{2}, x_{6}, x_{8}, x_{9}$.

Proof. Using the relations $f_{i}=0$ for $i=1, \ldots, 27$ we write down $9 \times$ 9-matrices for the action of multiplication by $x_{i}$ on the claimed basis elements for $i \in\{2,3,4,5,6,8,9\}$. The matrices, as may be checked, commute pairwise and obey relations corresponding to $f_{1}, \ldots, f_{27}$. So the evident homomorphism from $k\left[s, t, x_{0}, \ldots, x_{9}\right] /\left(f_{1}, \ldots, f_{27}\right)$ to the free $k\left[s, t, x_{0}, x_{1}, x_{7}\right]$-module of rank 9 is an isomorphism. 


\begin{tabular}{r|c}
$i$ & $f_{i}$ \\
\hline 1 & $-3 \zeta^{2} x_{0} x_{4}-x_{1} x_{3}+x_{2}^{2}$ \\
2 & $-t x_{1}^{2}+3 \zeta x_{0} x_{5}+x_{2} x_{3}$ \\
3 & $-t x_{1} x_{2}-3 x_{0} x_{6}+x_{3}^{2}$ \\
5 & $\left(\zeta-\zeta^{2}\right) x_{0} x_{7}-x_{1} x_{5}+x_{2} x_{4}$ \\
6 & $\left(1-\zeta^{2}\right) x_{0} x_{8}-x_{1} x_{6}+x_{3} x_{4}$ \\
7 & $-\left(\zeta-\zeta^{2}\right) x_{0} x_{9}-t x_{1} x_{4}+x_{3} x_{5}$ \\
8 & $\zeta^{2} x_{1} x_{6}+\zeta x_{3} x_{4}+x_{2} x_{5}$ \\
9 & $1-\zeta^{2} x_{1} x_{8}-\frac{1-\zeta^{2}}{3} x_{2} x_{7}+x_{4}^{2}$ \\
10 & $1-\zeta$ \\
11 & $x_{1} x_{9}-\frac{1-\zeta}{3} x_{3} x_{7}+x_{4} x_{5}$ \\
12 & $-x_{3} x_{7}+(1-\zeta) x_{4} x_{5}+x_{2} x_{8}$ \\
13 & $-t x_{1} x_{7}+\left(1-\zeta^{2}\right) x_{5}^{2}+x_{2} x_{9}$ \\
14 & $-9 s x_{0}^{2}+\zeta t x_{1} x_{5}+\zeta^{2} t x_{2} x_{4}+x_{3} x_{6}$ \\
15 & $-3\left(1-\zeta^{2}\right) s x_{0} x_{1}-t x_{1} x_{7}+(1-\zeta) x_{5}^{2}+x_{3} x_{8}$ \\
16 & $-\zeta) s x_{0} x_{2}-t x_{2} x_{7}+\left(1-\zeta^{2}\right) t x_{4}^{2}+x_{3} x_{9}$ \\
17 & $3 \zeta s x_{0} x_{1}-x_{5}^{2}+x_{4} x_{6}$ \\
18 & $-(1-\zeta) s x_{1}^{2}-\zeta x_{5} x_{7}+x_{4} x_{8}$ \\
19 & $-\left(1-\zeta^{2}\right) s x_{1} x_{2}-\zeta^{2} x_{6} x_{7}+x_{4} x_{9}$ \\
20 & $3 \zeta^{2} s x_{0} x_{2}-t x_{4}^{2}+x_{5} x_{6}$ \\
21 & $-(1-\zeta) s x_{1} x_{2}-\zeta x_{6} x_{7}+x_{5} x_{8}$ \\
22 & $-\left(1-\zeta^{2}\right) s x_{2}^{2}-\zeta^{2} t x_{4} x_{7}+x_{5} x_{9}$ \\
23 & $-3 s x_{0} x_{3}-t x_{4} x_{5}+x_{6}^{2}$ \\
24 & $-\left(1-\zeta^{2}\right) s x_{1} x_{3}+\left(\zeta-\zeta^{2}\right) s x_{2}^{2}-\zeta t x_{4} x_{7}+x_{6} x_{8}$ \\
25 & $-(1-\zeta) s x_{2} x_{3}-\zeta t x_{4} x_{8}+x_{6} x_{9}$ \\
26 & $-3 \zeta s x_{1} x_{4}-x_{7} x_{9}+x_{8}^{2}$ \\
27 & $-3 \zeta^{2} s x_{1} x_{5}-3 \zeta s x_{2} x_{4}-t x_{7}^{2}+x_{8} x_{9}$ \\
& $-3 \zeta^{2} s x_{2} x_{5}-t x_{7} x_{8}+x_{9}^{2}$ \\
\hline & $-30 x_{1}$
\end{tabular}

TABLE 1 . The polynomials $f_{1}, \ldots, f_{27} \in k\left[s, t, x_{0}, \ldots, x_{9}\right]$.

Lemma 9.1 implies that the ideal $\left(f_{1}, \ldots, f_{27}\right)$ of $k\left[s, t, x_{0}, \ldots, x_{9}\right]$ is prime. Indeed, the ideal is saturated with respect to $x_{0}, \ldots, x_{9}$ (e.g., $x_{0} g \in\left(f_{1}, \ldots, f_{27}\right)$ implies $g \in\left(f_{1}, \ldots, f_{27}\right)$ for $\left.g \in k\left[s, t, x_{0}, \ldots, x_{9}\right]\right)$, and the ideal generated by $f_{1}, \ldots, f_{27}$ in $k(s, t)\left[x_{0}, \ldots, x_{9}\right]$ defines an anticanonically embedded Brauer-Severi variety. So the latter ideal, and hence also the former ideal, is prime.

Proposition 9.2. Let $k$ be a field of characteristic different from 3 with primitive cube root of unity $\zeta, \pi: X \rightarrow \mathbb{A}^{2} \backslash\{0\}$ and $\pi^{\prime}: X^{\prime} \rightarrow \mathbb{A}^{2} \backslash\{0\}$ as in (9.1)-(9.2) and $f_{1}, \ldots, f_{27} \in k\left[s, t, x_{0}, \ldots, x_{9}\right]$ as in Table 1. The 
coherent sheaf $\pi_{*} \omega_{\pi}^{\vee}$ is free of rank 10 , and for some basis the schemetheoretic image in $\mathbb{A}^{2} \times \mathbb{P}^{9}$ is defined by the prime ideal $\left(f_{1}, \ldots, f_{27}\right)$ and is a nonsingular variety, flat over $\mathbb{A}^{2}$. For an explicit basis of the free coherent sheaf $\pi_{*}^{\prime} \omega_{\pi^{\prime}}^{\vee}$ of rank 10 the scheme-theoretic image in $\mathbb{A}^{2} \times \mathbb{P}^{9}$ is defined by the ideal obtained from $\left(f_{1}, \ldots, f_{27}\right)$ by applying $s \mapsto s^{3}$ and $t \mapsto t^{3}$, which is also prime. The fiber over $s=t=0$ is an irreducible, nonreduced scheme whose reduced subscheme is a cone over a twisted cubic curve.

The explicit basis mentioned in the statement of Proposition 9.2 is the one given above, as the basis of $\mathcal{S} \cap \mathcal{T}$.

Proof. As remarked after Lemma 9.1, the ideal generated by $f_{1}, \ldots, f_{27}$ is prime, and the same holds after applying $s \mapsto s^{3}$ and $t \mapsto t^{3}$. These facts, plus the remark about $\operatorname{Proj}\left(k(s, t)\left[x_{0}, \ldots, x_{9}\right] /\left(f_{1}, \ldots, f_{27}\right)\right)$ made before Lemma 9.1, justify the statements about scheme-theoretic image.

We directly analyze the fiber over $s=t=0$. First, the reduced subscheme is contained in the locus $x_{3}=x_{5}=x_{6}=x_{8}=x_{9}=0$, as we see directly from the defining equations. The defining equations with

$$
s=t=x_{3}=x_{5}=x_{6}=x_{8}=x_{9}=0
$$

no longer involve $x_{1}$. There are three equations, quadratic in $x_{0}, x_{2}, x_{4}$, $x_{7}$, the equations of a twisted cubic. So the reduced subscheme of the fiber is a cone over a twisted cubic. The coordinate point

$$
p_{0}=(1: 0: 0: 0: 0: 0: 0: 0: 0: 0)
$$

lies on the fiber and has Zariski tangent space of dimension 3. But $p_{0}$ is smooth point of the reduced subscheme. So, the fiber is nonreduced.

Now we consider the variety

$$
\operatorname{Proj}\left(k\left[s, t, x_{0}, \ldots, x_{9}\right] /\left(f_{1}, \ldots, f_{27}\right)\right) .
$$

Flatness over $\mathbb{A}^{2}$ follows from Lemma 9.1. It remains to show that this is a nonsingular variety. For this, we let the two-dimensional torus $\mathbb{G}_{m}^{2}$ act on $\mathbb{A}^{2}$ by $(\delta, \varepsilon) \cdot(s, t)=\left(\delta^{3} s, \varepsilon^{3} t\right)$ and on $\mathbb{P}^{9}$ by sending $\left(x_{0}: \cdots: x_{9}\right)$ to

$\left(\varepsilon^{3} x_{0}: \delta \varepsilon x_{1}: \delta \varepsilon^{2} x_{2}: \delta \varepsilon^{3} x_{3}: \delta^{2} \varepsilon x_{4}: \delta^{2} \varepsilon^{2} x_{5}: \delta^{2} \varepsilon^{3} x_{6}: \delta^{3} x_{7}: \delta^{3} \varepsilon x_{8}: \delta^{3} \varepsilon^{2} x_{9}\right)$

and thereby obtain a torus action on the variety defined by $f_{1}, \ldots, f_{27}$. Suppose the variety is singular. Since the singular locus is closed and torus-invariant, it must contain a fixed point, i.e., a coordinate point, of the fiber over $s=t=0$. From the description above, we only have to consider the coordinate points $p_{0}, p_{1}$, and $p_{7}$. For each we specify 7 
defining equations and 7 variables:

\begin{tabular}{c|c|c} 
point & equations & variables \\
\hline$p_{0}$ & $f_{1}, f_{2}, f_{3}, f_{4}, f_{5}, f_{6}, f_{13}$ & $s, x_{4}, x_{5}, x_{6}, x_{7}, x_{8}, x_{9}$ \\
$p_{1}$ & $f_{1}, f_{2}, f_{4}, f_{5}, f_{8}, f_{9}, f_{17}$ & $s, t, x_{3}, x_{5}, x_{6}, x_{8}, x_{9}$ \\
$p_{7}$ & $f_{4}, f_{8}, f_{9}, f_{17}, f_{18}, f_{25}, f_{26}$ & $t, x_{0}, x_{2}, x_{3}, x_{5}, x_{6}, x_{9}$
\end{tabular}

By direct computation we see that the corresponding Jacobian matrix at each $p_{i}$ is nonsingular, and thus $p_{i}$ is a nonsingular point of the variety defined by $f_{1}, \ldots, f_{27}$.

The proof of Theorem 1.2 proceeds as in the case of conic bundles [55]:

- Represent the generic fiber of $\pi$ by a 3-torsion Brauer group element $\alpha \in \operatorname{Br}(k(S))$. Apply embedded resolution of singularities so that $\alpha$ is ramified along a simple normal crossing divisor $D_{1} \cup \cdots \cup D_{\ell}$. Let $\mathcal{R}:=\sqrt[3]{\left(S,\left\{D_{1}, \ldots, D_{\ell}\right\}\right)}$ be the iterated root stack; then by Remark $2.4, \alpha$ is the restriction of an element

$$
\beta \in \operatorname{Br}(\mathcal{R}) \text {. }
$$

Pass to a $\mu_{3}$-gerbe $\mathcal{G} \rightarrow \mathcal{R}$ whose class is a lift $\beta_{0} \in H^{2}\left(\mathcal{R}, \mu_{3}\right)$ of $\beta$. The restriction of $\pi$ to $\pi^{-1}(U)$ is a smooth $\mathbb{P}^{2}$-bundle by [36, Lem. 1.7]. So, there is a corresponding sheaf of Azumaya algebras over $U$ and a locally free sheaf of rank 3 over $\mathcal{G} \times_{\mathcal{R}} U$. This spreads out to a coherent sheaf $\mathcal{E}$ on $\mathcal{G}$, which we may take to be reflexive, hence [33] only failing to be locally free, if at all, on a locus of codimension $\geq 3$.

- By a standard construction (closure in Grassmannian of rank 3 quotients of $\mathcal{E}$ ), followed by desingularization and destackification [14], we may suppose that $\mathcal{E}$ is locally free and $\mathcal{R}$ is an iterated root stack $\sqrt[3]{\left(\widetilde{S},\left\{\widetilde{D}_{1}, \ldots, \widetilde{D}_{m}\right\}\right)}$ over a smooth projective variety $\widetilde{S}$. We may suppose, for every $i$, that over a general point of $\widetilde{D}_{i}$ the stabilizer action on the fiber of $\mathbb{P}(\mathcal{E})$ is balanced, since otherwise it may be made trivial with suitable elementary transformations.

- After blowing up intersections of pairs and triples of divisors, we may arrange that no triple of divisors intersects, and for every pair of intersecting divisors, the corresponding projective representation $\mu_{3} \times \mu_{3} \rightarrow P G L_{3}$ over a general point of the intersection is faithful. The group-theoretic input to these assertions is the fact that no more than two independent commuting copies of $\mu_{3}$ may be found in $P G L_{3}$.

- Apply Proposition 4.4 to the complement of the intersections of pairs of divisors, and Propositions 8.1 and 9.2 and Lemma 2.8 to fill in the fibers over intersections of divisors. 


\section{Applichtion to Rationality}

Here we prove Theorem 1.4. We use a theorem of Voisin [64, Thm. $2.1]$, in the form of [21, Thm. 2.3]:

Theorem 10.1. Let $k$ be an uncountable algebraically closed field, $B$ a variety over $k$, and $\phi: \mathcal{X} \rightarrow B$ a flat projective morphism with integral fibers and smooth generic fiber. Suppose that there exists a $b_{0} \in B$ such that the fiber $X_{0}:=\phi^{-1}\left(b_{0}\right)$ satisfies:

(O) for some positive integer $n$, invertible in $k$, there is nontrivial unramified cohomology of $\mu_{n}$ on $X_{0}$;

(R) $X_{0}$ admits a universally $\mathrm{CH}_{0}$-trivial resolution of singularities.

Then, for very general $b \in B$, the fiber $X_{b}:=\phi^{-1}(b)$ is not stably rational.

The reader may turn to [21] for the definitions of universally $\mathrm{CH}_{0^{-}}$ trivial scheme and universally $\mathrm{CH}_{0}$-trivial morphism. For a proper geometrically integral scheme $X$ over a field $\ell$, say of dimension $d$, a related notion (equivalent, when $X$ is smooth [21, Prop. 1.4]), is for $X$ to admit a Chow decomposition of the diagonal. This means that in the Chow group $\mathrm{CH}_{d}(X \times X)$ of $d$-dimensional cycles modulo rational equivalence, the class $\left[\Delta_{X}\right]$ of the diagonal of $X$ is equal to a sum

$$
Z+[X] \times z_{0}
$$

for some $d$-dimensional cycle $Z$ supported on $D \times X$, for a closed subset $D \subsetneq X$, and 0 -cycle $z_{0}$ of degree 1 on $X$. If we let $\delta_{X} \in \mathrm{CH}_{0}\left(X_{\ell(X)}\right)$ denote the restriction of $\Delta_{X}$ to $X_{\ell(X)}:=\operatorname{Spec}(\ell(X)) \times_{\operatorname{Spec}(\ell)} X$, this is equivalent to:

(*) $\quad X$ has a 0 -cycle $z_{0}$ of degree 1 and $\left[\delta_{X}\right]=z_{0}$ in $\mathrm{CH}_{0}\left(X_{\ell(X)}\right)$.

Condition $(\mathrm{R})$, with $\mathrm{CH}_{0}$-trivial resolution of singularities $\widetilde{X}_{0} \rightarrow X_{0}$, implies the equivalence of $(*)$ on $X_{0}$ and $\widetilde{X}_{0}$, hence the equivalence of admitting a Chow decomposition of the diagonal. Chow decomposition of the diagonal is obstructed by Condition (O) but implied by stable rationality, while an argument with Chow schemes [21, App. B] (Chow functors in case $\operatorname{char}(k)>0[34, \S 3])$ shows that the $k$-points whose fibers admit a Chow decomposition of the diagonal occupy a countable union of closed subsets of $B$. An alternative approach, bypassing Chow groups, appears in recent work [54], [40].

We return to Theorem 1.4, with $\operatorname{char}(k) \neq 2,3$, and smooth degree 2 del Pezzo surface $S$.

We first treat the case $d=3$ and then explain the modifications to deal with general $d$. The anticanonical morphism expresses $S$ as double 
cover of $\mathbb{P}^{2}$ branched along a nonsingular quartic curve $R$, which we take to have defining equation $h$ with respect to some coordinates.

Step 1. There exists a nonsingular cubic curve $D_{0}$ meeting $R$ in six tangencies lying on a conic. Among the 28 bitangent lines to $R$, at most 12 are hyperflexes, since the weighted count of Weierstrass points of $R$ is 24 and each hyperflex contributes 2; cf. [62]. Bitangent lines are identified with odd theta characteristics, and it is known classically (see, e.g., $[26, \S 2])$ that any set of 8 bitangent lines, which we may suppose disjoint from the set of hyperflexes, contains a syzygetic triple $\ell, \ell^{\prime}, \ell^{\prime \prime}$ : the six points of intersection with $R$ lie on a conic $f=0$. Using the same symbols for defining linear forms and suitably rescaling $h$, we obtain by Max Noether's theorem a relation $h=f^{2}+\ell \ell^{\prime} \ell^{\prime \prime} \ell^{\prime \prime \prime}$ for a fourth bitangent $\ell^{\prime \prime \prime}$. Let $j$ be a line in general position with respect to $\ell, \ell^{\prime}, \ell^{\prime \prime}$ with suitably scaled defining equation. Then we easily see that the cubic $g:=\ell \ell^{\prime} \ell^{\prime \prime}-2 f j-j^{2} \ell^{\prime \prime \prime}$, appearing in the relation $h=\left(f+j \ell^{\prime \prime \prime}\right)^{2}+g \ell^{\prime \prime \prime}$, is nonsingular and meets $R$ in six tangencies.

Step 2. Let $\widetilde{D}_{0} \rightarrow D_{0}$ be a nontrivial cyclic degree 3 étale cover, determining (Remark 2.3) a 3-torsion element of $\operatorname{Br}\left(\mathbb{P}^{2} \backslash D_{0}\right)$, which extends (Remark 2.4) to an element of $\operatorname{Br}\left(\sqrt[3]{\left(\mathbb{P}^{2}, D_{0}\right)}\right)$, represented $([7]$, [23]) by an index 3 division algebra at the generic point. As in the proof of Theorem 1.2, it spreads out to a sheaf of Azumaya algebras over $\sqrt[3]{\left(\mathbb{P}^{2}, D_{0}\right)}$. We obtain an element $\gamma_{0} \in H^{2}\left(\sqrt[3]{\left(\mathbb{P}^{2}, D_{0}\right)}, \mu_{3}\right)$, gerbe $G_{0} \rightarrow \sqrt[3]{\left(\mathbb{P}^{2}, D_{0}\right)}$ with this class, and locally free coherent sheaf $E_{0}$ of rank 3 on $G_{0}$ such that the pullback of the sheaf of Azumaya algebras is $\operatorname{End}\left(E_{0}\right)$. We perform base change via $S \rightarrow \mathbb{P}^{2}$ to obtain reducible curve $D=D_{1} \cup D_{2}$ with étale degree 3 cover $\widetilde{D} \rightarrow D$, element $\gamma \in$ $H^{2}\left(\sqrt[3]{(S, D)}, \mu_{3}\right)$, gerbe $G \rightarrow \sqrt[3]{(S, D)}$, and locally free coherent sheaf $E$ on $G$.

Step 3. Apply the deformation-theoretic machinery of $[34, \S 4.3]$ to exhibit:

- an exact sequence $0 \rightarrow \widetilde{E} \rightarrow E \rightarrow Q \rightarrow 0$, where $Q$ is supported on a divisor and is locally free there of rank 2 , such that the space of obstructions for $\widetilde{E}$ vanishes.

- an étale neighborhood $B$ of the point $b_{0}$, corresponding to divisor $D$ and cyclic cover $\widetilde{D}$, in the space of reduced nodal curves on $S$ with cyclic degree 3 étale cover, corresponding divisor $\mathcal{D} \subset$ $B \times S$, root stack $\sqrt[3]{(B \times S, \mathcal{D})}$, class $\Gamma \in H^{2}\left(\sqrt[3]{(B \times S, \mathcal{D})}, \mu_{3}\right)$ restricting to $\gamma$, gerbe $\mathcal{G} \rightarrow \sqrt[3]{(B \times S, \mathcal{D})}$ restricting to $G$, locally free coherent sheaf $\widetilde{\mathcal{E}}$ restricting to $\widetilde{E}$. 
- a smooth $\mathbb{P}^{2}$-bundle $P \rightarrow \sqrt[3]{(B \times S, \mathcal{D})}$, which upon base change to $\mathcal{G}$ yields $\mathbb{P}(\widetilde{\mathcal{E}})$.

Step 4. Apply Proposition 4.4 to $P$ to obtain a Brauer-Severi bundle $\pi: \mathcal{X} \rightarrow B \times S$ satisfying the hypotheses of Theorem 10.1. Since, by construction, the discriminant curve of the Brauer-Severi bundle $X_{0} \rightarrow$ $\left\{b_{0}\right\} \times S$ has two components and the Brauer class is given by cyclic covers, nontrivial on each component and étale over the nodes, condition $(\mathrm{O})$ is satisfied. Condition $(\mathrm{R})$ holds, since the singularities of $X_{0}$ (which lie over the nodes of the discriminant curve) are of toric type.

The case $d>3$ is dealt with by taking $D_{3}$ to be the pre-image in $S$ of a general curve $C \subset \mathbb{P}^{2}$ of degree $d-3$ and defining $D_{0}^{\prime}:=D_{0} \cup C$. We pull back $\gamma_{0} \in H^{2}\left(\sqrt[3]{\left(\mathbb{P}^{2}, D_{0}\right)}, \mu_{3}\right)$ and the gerbe $G_{0}$ to obtain $\gamma_{0}^{\prime} \in$ $H^{2}\left(\sqrt[3]{\left(\mathbb{P}^{2}, D_{0}^{\prime}\right)}, \mu_{3}\right)$ and a gerbe $G_{0}^{\prime}$. The pull-back of the locally free coherent sheaf $E_{0}$ has trivial $\mu_{3}$-stabilizer actions over points of $C$. By performing suitable elementary transformations over the component of the gerbe of the root stack over $C$, we may obtain balanced actions, compatible with those over $D_{0}$. Then the smooth $\mathbb{P}^{2}$-bundle corresponding to the locally free sheaf has the same étale local isomorphism type as that corresponding to $E$ (Step 2). We thereby obtain an extension of $\gamma_{0}^{\prime}$ to $\gamma_{0}^{\prime \prime} \in H^{2}\left(\sqrt[3]{\left(\mathbb{P}^{2}, D_{0}^{\prime}\right)}, \mu_{3}\right)$, of $G_{0}^{\prime}$ to $G_{0}^{\prime \prime} \rightarrow \sqrt[3]{\left(\mathbb{P}^{2}, D_{0}^{\prime}\right)}$, and of $E$ to a locally free coherent sheaf on $G_{0}^{\prime \prime}$. We proceed with Steps 3 and 4 , taking $D$ to be the union of $D_{1}, D_{2}$, and the pull-back $D_{3}$ of $C$; the triviality of the cyclic cover over $D_{3}$ poses no problems in carrying out these steps.

\section{Appendix A. Birational contractions}

Let $k$ be a field and $X$ a scheme, proper over $k$. A line bundle $L$ on $X$ is called semiample if $L^{m}$ is globally generated for some $m>0$. Then the set $M(X, L)$ of $m \geq 0$, such that $L^{m}$ is globally generated, consists, for $m \gg 0$, of precisely the multiples of a well-defined positive integer, the exponent of $M(X, L)$; cf. [44, §2.1.B]. We start by recalling the contraction determined by a semiample line bundle.

Proposition A.1. Let $X$ be a scheme, proper over a field $k$, with semiample line bundle $L$ and sections $s_{0}, \ldots, s_{M} \in H^{0}\left(X, L^{m}\right)$ generating $L^{m}$ for some $m>0$, thereby inducing a morphism $\psi: X \rightarrow \mathbb{P}^{M}$. Let $Y=\psi(X)$, and write the Stein factorization of $\psi$ as morphism

$$
\pi: X \rightarrow X^{\prime}
$$

followed by finite morphism $f: X^{\prime}=\operatorname{Spec}\left(\psi_{*} \mathcal{O}_{X}\right) \rightarrow Y$. We let $\mathcal{O}_{Y}(1)$, respectively $\mathcal{O}_{X^{\prime}}(1)$ denote the restriction to $Y$, respectively the pull-back to $X^{\prime}$ of the line bundle $\mathcal{O}_{\mathbb{P}^{M}}(1)$, so $\pi^{*} \mathcal{O}_{X^{\prime}}(1) \cong L^{m}$. Then: 
(i) The morphism $\pi: X \rightarrow X^{\prime}$ is proper and induces an isomorphism $\mathcal{O}_{X^{\prime}} \stackrel{\sim}{\rightarrow} \pi_{*} \mathcal{O}_{X}$.

(ii) For all $n \gg 0$ the line bundle $\mathcal{O}_{X^{\prime}}(n)$ is very ample, and for any positive integer $n$ such that $\mathcal{O}_{X^{\prime}}(n)$ is very ample, the morphism $X \rightarrow \mathbb{P}^{N}$ determined by a basis of $H^{0}\left(X, L^{m n}\right)$ factors uniquely through $X^{\prime}$, identifying $X^{\prime}$ with the image of $X$ in $\mathbb{P}^{N}$.

(iii) With the property in (ii) the scheme $X^{\prime}$, with $\pi: X \rightarrow X^{\prime}$, is determined uniquely up to canonical isomorphism, independently of the choice of $m$ and the generating sections, and the powers of $L$ that are isomorphic to the pull-back of a line bundle from $X^{\prime}$ are precisely all multiples of the exponent of $M(X, L)$.

Proof. We have stated, in (i), a basic property of the Stein factorization; see [28, 4.3.1]. The first statement in (ii) follows from standard facts on very ample line bundles $[27,4.4 .10(\mathrm{ii}), 5.1 .6]$, and for the second statement, using the isomorphism $\mathcal{O}_{X^{\prime}} \cong \pi_{*} \mathcal{O}_{X}$ we have

$$
H^{0}\left(X, L^{m n}\right) \cong H^{0}\left(X^{\prime}, \mathcal{O}_{X^{\prime}}(n)\right)
$$

so for $n$ such that $\mathcal{O}_{X^{\prime}}(n)$ is very ample, a choice of basis compatibly maps $X$ to $\mathbb{P}^{N}$ and embeds $X^{\prime}$ in $\mathbb{P}^{N}$. The factorization is unique, and by (i), identifies $X^{\prime}$ with the image of $X \rightarrow \mathbb{P}^{N}$. For (iii), if $\tilde{s}_{0}, \ldots, \tilde{s}_{\widetilde{M}}$ is another collection of generating sections of $L^{m}$, leading to $\tilde{\pi}: X \rightarrow \widetilde{X}^{\prime}$, then for $n$ such that $\mathcal{O}_{X^{\prime}}(n)$ and $\mathcal{O}_{\tilde{X}^{\prime}}(n)$ are very ample, $X^{\prime}$ and $\tilde{X}^{\prime}$ are both identified with the image of $X \rightarrow \mathbb{P}^{N}$, as in (ii). The isomorphism $X^{\prime} \cong \tilde{X}^{\prime}$ obtained this way is independent of $n$ : it suffices to compare the isomorphisms arising from $n$, leading to $X \rightarrow \mathbb{P}^{N}$, and $d n$, for some $d>0$, leading to $X \rightarrow \mathbb{P}^{N^{\prime}}$, but these are compatible with the $d$-tuple Veronese embedding of $\mathbb{P}^{N}$ and the rational map from $\mathbb{P}^{N^{\prime}}$ corresponding to

$$
\operatorname{Sym}^{d}\left(H^{0}\left(X, L^{m n}\right)\right) \rightarrow H^{0}\left(X, L^{d m n}\right) .
$$

When $\pi: X \rightarrow X^{\prime}$ and $\tilde{\pi}: X \rightarrow \tilde{X}^{\prime}$ arise from different powers of $L$ the argument is similar, with pairs $n$ and $\tilde{n}$ of positive integers chosen proportionally to yield the same power of $L$ in the respective isomorphisms (A.1) with $H^{0}\left(X^{\prime}, \mathcal{O}_{X^{\prime}}(n)\right)$ and $H^{0}\left(\widetilde{X}^{\prime}, \mathcal{O}_{\widetilde{X}^{\prime}}(\tilde{n})\right)$. Taking, as powers of $L$, two sufficiently large consecutive multiples of the exponent of $M(X, L)$ yields respective line bundles $\mathcal{O}_{X^{\prime}}(1)$, such that the pull-back of their difference is isomorphic to $L^{d}$, where $d$ denotes the exponent of $M(X, L)$. If a line bundle $L^{\prime}$ on $X^{\prime}$ satisfies $\pi^{*} L^{\prime} \cong L^{e}$ for some $e>0$ then $L^{\prime}$ is ample, so all sufficiently large powers of $L^{\prime}$ are globally generated and thus $e$ is divisible by $d$. 
A morphism satisfying the properties stated in (i) is called a contraction and is necessarily surjective with geometrically connected fibers [28, 4.3.2, 4.3.4]. We call this the contraction determined by $L$.

Below we present a result which encompasses the following examples.

Example A.2. We suppose $X$ is smooth and projective over $k$ of dimension $n$, with ample line bundle $L_{0}$, and effective divisor $E \subset X$.

(i) If $E \cong \mathbb{P}^{n-1}$ with normal bundle $N_{E / X} \cong \mathcal{O}_{\mathbb{P}^{n-1}}(-1)$, and $m$ denotes the positive integer with $\left.L_{0}\right|_{E} \cong \mathcal{O}_{\mathbb{P}^{n-1}}(m)$, then $L:=$ $L_{0}(m E)$ is semiample and determines a contraction

$$
X \cong B \ell_{\{p\}} X^{\prime} \rightarrow X^{\prime}
$$

of $E$ to a $k$-point $p$ on a nonsingular projective variety $X^{\prime}$.

(ii) If $n=3$ and $E \cong \mathbb{P}^{1} \times \mathbb{P}^{1}$, with $N_{E / X} \cong \mathcal{O}_{\mathbb{P}^{1} \times \mathbb{P}^{1}}(-1,-1)$ and $\left.L_{0}\right|_{E} \cong \mathcal{O}_{\mathbb{P}^{1} \times \mathbb{P}^{1}}\left(m_{1}, m_{2}\right)$, then $L:=L_{0}\left(\min \left(m_{1}, m_{2}\right) E\right)$ is semiample and determines a contraction

$$
X \cong B \ell_{C} X^{\prime} \rightarrow X^{\prime}
$$

for a curve $C \cong \mathbb{P}^{1}$ on nonsingular $X^{\prime}$ with $N_{C / X^{\prime}} \cong \mathcal{O}_{\mathbb{P}^{1}}(-1)^{2}$, when $m_{1} \neq m_{2}$, respectively

$$
X \cong B \ell_{\{p\}} X^{\prime} \rightarrow X^{\prime}
$$

for a $k$-point $p \in X^{\prime}$ with an ordinary double point singularity, when $m_{1}=m_{2}$.

When $\operatorname{dim}(X)=2$, Example A.2(i) is the contraction theorem of Castelnuovo and Enriques and leads to minimal models of algebraic surfaces. The case of dimension at least 3 requires the sophistication of the minimal model program (see [37] for a survey), including the observation that a curve class (modulo numerical equivalence), which meets a canonical divisor positively and is extremal in the cone of effective classes, uniquely determines a contraction. Mori, in dimension 3, identifies five kinds of divisorial contractions [51, Thm. 3.3], including the ones given in Example A.2; we see already the possibility of contraction to a singular point, which is essential for the theory. The results below on divisorial contractions to a point (Proposition A.3) and to a general subscheme (Proposition A.7) are essentially contained in [51, Lem. 3.32]; a relative version is presented in Proposition A.9.

An important ingredient, already appearing in loc. cit., is the following notion [52]: an ample line bundle $L$ on a projective scheme $X$ is normally generated if

$$
\operatorname{Sym}^{n} H^{0}(X, L) \rightarrow H^{0}\left(X, L^{n}\right)
$$

is surjective for every positive integer $n$. 
Proposition A.3. Let $X$ be a projective scheme over $k$ with ample line bundle $L_{0}$ and effective Cartier divisor $E \subset X$ with $H^{0}\left(E, \mathcal{O}_{E}\right)=k$ (this is the case, e.g., when geometrically $E$ is connected and reduced). Suppose that $N_{E / X}^{\vee}$ is ample, with $\left.L_{0}\right|_{E} \otimes\left(N_{E / X}\right)^{m}$ a torsion line bundle for some $m>0$. Assume, furthermore, that $N_{E / X}^{\vee}$ is normally generated, and $H^{1}\left(E,\left(N_{E / X}^{\vee}\right)^{n}\right)=0$ for all $n>0$. Then $L:=L_{0}(m E)$ is semiample with exponent of $M(X, L)$ equal to the order of $\left.L_{0}\right|_{E} \otimes\left(N_{E / X}\right)^{m}$ in $\operatorname{Pic}(E)$ and determines a contraction $X \cong B \ell_{\{p\}} Y \rightarrow Y$ of $E$ to a k-point $p \in Y$ such that the local ring $\mathcal{O}_{Y, p}$ has associated graded $k$-algebra isomorphic to $\bigoplus H^{0}\left(E,\left(N_{E / X}^{\vee}\right)^{n}\right)$.

Proof. Replacing $L_{0}$ by some power, we may suppose $L_{0}$ is very ample with $H^{1}\left(X, L_{0}\right)=0$, the restriction of $L$ to $E$ is trivial, and $m \geq 2$. Now, we claim, $H^{1}\left(X, L_{0}(j E)\right)=0$ for $0 \leq j<m$. We have this for $j=0$, and we argue by induction on $j$ using the exact sequence in cohomology associated with

$$
0 \rightarrow L_{0}((j-1) E) \rightarrow L_{0}(j E) \rightarrow\left(N_{E / X}^{\vee}\right)^{m-j} \rightarrow 0 .
$$

Then, by considering the sequence for $j=m$ we find that $L$ has a global section which restricts to a nonzero constant on $E$ (under $\left.L\right|_{E} \cong \mathcal{O}_{E}$ ). Furthermore, by the sequence for $j=m-1$ we may lift a basis of $H^{0}\left(E, N_{E / X}^{\vee}\right)$ to global sections of $L$, vanishing along $E$. We may view, as well, all the global sections of $L_{0}$ as global sections of $L$. So we see that $L$ is globally generated, and the induced morphism to its image $Y$ in projective space collapses $E$ to a point $p$, such that the scheme-theoretic pre-image of $p$ is $E$, and $X \backslash E \cong Y \backslash\{p\}$. Let us denote by $\mathcal{I}_{E}$ the ideal sheaf of $E$, so $\mathcal{I}_{E} / \mathcal{I}_{E}^{2} \cong N_{E / X}^{\vee}$. Then we see from these two cases of the sequence,

$$
\mathfrak{m}_{p} / \mathfrak{m}_{p}^{2} \rightarrow H^{0}\left(E, \mathcal{I}_{E} / \mathcal{I}_{E}^{2}\right)
$$

is surjective. Again replacing $L_{0}$ with a tensor power we may suppose that the morphism $\pi: X \rightarrow Y$ given by the global sections of $L$ induces an isomorphism $\mathcal{O}_{Y} \stackrel{\sim}{\rightarrow} \pi_{*} \mathcal{O}_{X}$. Then by the Theorem on Formal Functions, we have an isomorphism

$$
\widehat{\mathcal{O}}_{Y, p} \stackrel{\sim}{\rightarrow} \lim _{\longleftarrow} H^{0}\left(X, \mathcal{O}_{X} / \mathcal{I}_{E}^{n}\right) .
$$

We claim, the isomorphism (A.2) comes from isomorphisms

$$
\mathcal{O}_{Y, p} / \mathfrak{m}_{p}^{n} \rightarrow H^{0}\left(X, \mathcal{O}_{X} / \mathcal{I}_{E}^{n}\right)
$$

for every $n$.

We begin by observing, using the $H^{1}$-vanishing hypothesis, that

$$
H^{0}\left(X, \mathcal{I}_{E}^{i} / \mathcal{I}_{E}^{j+1}\right) \rightarrow H^{0}\left(X, \mathcal{I}_{E}^{i} / \mathcal{I}_{E}^{j}\right)
$$


is surjective for all $i<j$. We will use this fact several times in the remainder of the proof.

By the normal generation hypothesis, for every $j$, the homomorphism

$$
H^{0}\left(E, \mathcal{I}_{E} / \mathcal{I}_{E}^{2}\right) \otimes H^{0}\left(E, \mathcal{I}_{E}^{j-1} / \mathcal{I}_{E}^{j}\right) \rightarrow H^{0}\left(E, \mathcal{I}_{E}^{j} / \mathcal{I}_{E}^{j+1}\right),
$$

given by multiplication, is surjective. Combining this with the observation about $\mathfrak{m}_{p} / \mathfrak{m}_{p}^{2}$, we have the surjectivity of

$$
\mathfrak{m}_{p} / \mathfrak{m}_{p}^{2} \otimes H^{0}\left(E, \mathcal{I}_{E}^{j-1} / \mathcal{I}_{E}^{j}\right) \rightarrow H^{0}\left(E, \mathcal{I}_{E}^{j} / \mathcal{I}_{E}^{j+1}\right) .
$$

We see more generally that if $s_{1}, \ldots, s_{\ell}$ denote lifts of a basis of $\mathfrak{m}_{p} / \mathfrak{m}_{p}^{2}$ to the coordinate ring of an affine neighborhood of $p$, then multiplication by $s_{1}, \ldots, s_{\ell}$ induces surjective

$$
H^{0}\left(X, \mathcal{I}_{E}^{i} / \mathcal{I}_{E}^{j}\right)^{\ell} \rightarrow H^{0}\left(X, \mathcal{I}_{E}^{i+1} / \mathcal{I}_{E}^{j+1}\right)
$$

for all $i<j$. Indeed, we have the exact sequences

$$
0 \rightarrow H^{0}\left(X, \mathcal{I}_{E}^{j-1} / \mathcal{I}_{E}^{j}\right)^{\ell} \rightarrow H^{0}\left(X, \mathcal{I}_{E}^{i} / \mathcal{I}_{E}^{j}\right)^{\ell} \rightarrow H^{0}\left(X, \mathcal{I}_{E}^{i} / \mathcal{I}_{E}^{j-1}\right)^{\ell} \rightarrow 0
$$

and

$$
0 \rightarrow H^{0}\left(X, \mathcal{I}_{E}^{j} / \mathcal{I}_{E}^{j+1}\right) \rightarrow H^{0}\left(X, \mathcal{I}_{E}^{i+1} / \mathcal{I}_{E}^{j+1}\right) \rightarrow H^{0}\left(X, \mathcal{I}_{E}^{i+1} / \mathcal{I}_{E}^{j}\right) \rightarrow 0
$$

with compatible vertical maps given by multiplication with $s_{1}, \ldots, s_{\ell}$. Induction on $j$ for each fixed $i$ establishes the surjectivity of (A.4).

If we regard $i$ as fixed and apply to (A.4) the general fact that an inverse system of surjective linear maps of finite-dimensional vector spaces induces a surjective map of inverse limits, then we find,

$$
\mathfrak{m}_{p} \lim _{\longleftarrow} H^{0}\left(X, \mathcal{I}_{E}^{i} / \mathcal{I}_{E}^{n}\right)=\lim _{\longleftarrow} H^{0}\left(X, \mathcal{I}_{E}^{i+1} / \mathcal{I}_{E}^{n}\right) .
$$

An inductive argument now shows that we have an isomorphism

$$
\mathfrak{m}_{p}^{i} \widehat{\mathcal{O}}_{Y, p} \stackrel{\sim}{\rightarrow} \underset{\varliminf}{\lim } H^{0}\left(X, \mathcal{I}_{E}^{i} / \mathcal{I}_{E}^{n}\right),
$$

for every $i$. The case $i=0$ is (A.2), and from (A.8) we have an isomorphism of the respective ideals generated on each side by $\mathfrak{m}_{p}$, which with (A.7) gives us the inductive step.

The claim is now immediate from (A.2) and (A.8). Notice that the claim implies that for every $n$ we have an isomorphism

$$
\mathfrak{m}_{p}^{n-1} / \mathfrak{m}_{p}^{n} \rightarrow H^{0}\left(E, \mathcal{I}_{E}^{n-1} / \mathcal{I}_{E}^{n}\right) .
$$

The statement concerning the associated graded ring of $\mathcal{O}_{Y, p}$ in the proposition is immediate from (A.9). By the universal property of blowing up, 
we obtain a unique morphism $X \rightarrow B \ell_{\{p\}} Y$ factorizing $\pi$, whose restriction to $E$ is given by

$$
E \cong \operatorname{Proj}\left(\bigoplus H^{0}\left(E, \mathcal{I}_{E}^{n} / \mathcal{I}_{E}^{n+1}\right)\right) \stackrel{\sim}{\rightarrow} \operatorname{Proj}\left(\bigoplus \mathfrak{m}_{p}^{n} / \mathfrak{m}_{p}^{n+1}\right)
$$

Now $X \rightarrow B \ell_{\{p\}} Y$ is a morphism of projective schemes, such that the scheme-theoretic pre-image of every point of $B \ell_{\{p\}} Y$ is a reduced point with the same residue field. By [29, 18.12.6], this must be a closed immersion. But the exceptional divisor of the blow-up is an effective Cartier divisor, over the complement of which we have an isomorphism, hence $X \rightarrow B \ell_{\{p\}} Y$ is an isomorphism.

For the version of Proposition A.3 which allows contraction to a more general variety than a point, we need relative versions of the hypotheses. A relative version of the hypothesis $H^{0}\left(E, \mathcal{O}_{E}\right)=k$ is to be given a flat contraction morphism $E \rightarrow F$, and the conclusion will be that this is determined by $\left.L\right|_{E}$.

Lemma A.4. Let $Y=\operatorname{Spec}(A)$ be a Noetherian affine scheme, $\pi: X \rightarrow$ $Y$ a flat projective morphism, $L$ an ample line bundle on $X$, and $y \in Y$ a point such that the restriction $L_{y}$ of $L$ to the fiber $X_{y}$ is very ample (resp. is normally generated) and satisfies $H^{1}\left(X_{y}, L_{y}^{n}\right)=0$, for $n=1$ (resp. for all $n>0)$. Then there exists an open subscheme $U \subset Y$ with $y \in U$ such that relative to $X \times_{Y} U \rightarrow U$ the restriction of $L$ is very ample (resp. is very ample with

$$
\operatorname{Sym}^{n} H^{0}\left(X \times_{Y} U, L\right) \rightarrow H^{0}\left(X \times_{Y} U, L^{n}\right)
$$

surjective for all $n>0$ ).

Proof. By the machinery of cohomology and base change, we may replace $Y$ by a Zariski neighborhood of $y$, such that the coherent sheaf $\pi_{*} L$ is locally free and its formation commutes with arbitrary base change. Since $L_{y}$ is globally generated, the cokernel of $\pi^{*} \pi_{*} L \rightarrow L$ has trivial fiber over $y$, hence may be assumed to vanish if we shrink $Y$ further. We obtain a morphism $X \rightarrow \mathbb{P}\left(\left(\pi_{*} L\right)^{\vee}\right)$ over $Y$, and this restricts to a closed immersion of $X_{y}$ since $L_{y}$ is very ample. The support of the cokernel of $\mathcal{O}_{\mathbb{P}_{Y}^{N}} \rightarrow \mathcal{O}_{X}$ is disjoint from $X_{y}$, hence by further shrinking we find that $L$ is relatively very ample. There exists $n_{0}$ such that

$$
\operatorname{Sym}^{n} H^{0}(X, L) \rightarrow H^{0}\left(X, L^{n}\right)
$$

is surjective for all $n>n_{0}$ (consequence of the vanishing of $H^{1}$ of all sufficiently large twists of the ideal sheaf of $X$ in $\left.\mathbb{P}\left(H^{0}(X, L)^{\vee}\right)\right)$. Now suppose that $L_{y}$ is normally generated. Then, as above, we may suppose that $\pi_{*} L^{n}$ is locally free and its formation commutes with arbitrary base change for all $1 \leq n \leq n_{0}$. For all such $n$, we consider the cokernel 
of $\operatorname{Sym}^{n} H^{0}(X, L) \rightarrow H^{0}\left(X, L^{n}\right)$, which may be assumed to vanish by shrinking $Y$, and thus we have the desired conclusion when $L_{y}$ is normally generated.

Lemma A.5. Let $X$ be a scheme, with projective morphism $\pi: X \rightarrow S$ to a Noetherian scheme $S$ and relatively ample line bundles $L$ and $M$. Then for every coherent sheaf $\mathcal{F}$ on $X$ there exists $n \in \mathbb{N}$, such that $R^{i} \pi_{*}\left(\mathcal{F} \otimes L^{a} \otimes M^{b}\right)=0$ for all $i>0$ and $a, b \in \mathbb{N}$ with $\max (a, b) \geq n$.

Proof. We immediately reduce to the case $S$ when is affine. Using the ampleness of $L$ and $M$, we reduce further to establishing the assertion with $\max (a, b)$ replaced by $\min (a, b)$. By considering $\mathcal{F}$ twisted by various powers of $L$ and $M$ and replacing $L$ and $M$ with suitable powers, we may suppose that $L$ and $M$ are very ample. By pushing forward $\mathcal{F}$ along the closed immersion $X \rightarrow \mathbb{P}_{S}^{\ell} \times{ }_{S} \mathbb{P}_{S}^{m}$ for some $\ell, m \in \mathbb{N}$ determined by the sections of $L$ and $M$, we are reduced to proving the following assertion: if $\mathcal{F}$ is a coherent sheaf on $\mathbb{P}_{S}^{\ell} \times{ }_{S} \mathbb{P}_{S}^{m}$ then there exists $n \in \mathbb{N}$ such that

$$
H^{i}\left(\mathbb{P}_{S}^{\ell} \times{ }_{S} \mathbb{P}_{S}^{m}, \mathcal{F}(a, b)\right)=0 \quad \text { for all } a, b \in \mathbb{N} \text { with } \min (a, b) \geq n .
$$

Here, $\mathcal{F}(a, b)$ denotes the twist by the pullback of $\mathcal{O}_{\mathbb{P}_{S}^{\ell}}(a)$ and the pullback of $\mathcal{O}_{\mathbb{P}_{S}^{m}}(b)$. The proof is by descending induction on $i$, starting from the case $i=\ell+m+1$, which is trivial by the vanishing of cohomology in degrees greater than the relative dimension. For the inductive step, the ampleness of $\mathcal{O}_{\mathbb{P}_{S}^{\ell} \times{ }_{S} \mathbb{P}_{S}^{m}}(1,1)$ implies the existence of $c, d \in \mathbb{N}$ such that there is an exact sequence

$$
0 \rightarrow \mathcal{G} \rightarrow \mathcal{O}_{\mathbb{P}_{S}^{\ell} \times{ }_{S} \mathbb{P}_{S}^{m}}(-c,-c)^{\oplus d} \rightarrow \mathcal{F} \rightarrow 0
$$

of coherent sheaves. Twisting and applying the induction hypothesis and known cohomology of line bundles on $\mathbb{P}_{S}^{\ell} \times{ }_{S} \mathbb{P}_{S}^{m}$, we obtain the result.

Lemma A.6. Let $X$ be a scheme, with projective morphism $\pi: X \rightarrow S$ to a Noetherian scheme $S$ and line bundles $L$ and $M$, such that locally on $S$ the line bundle $L$ is semiample, determining a flat contraction $\psi: X \rightarrow$ $Y$, and $M$ is relatively ample. Suppose $c, d \in \mathbb{N}$ are such that for all $y \in Y$ the fiber $X_{y}$ satisfies $H^{i}\left(X_{y},\left(\left.M\right|_{X_{y}}\right)^{j}\right)=0$ for all $1 \leq i \leq c$ and $j \geq d$. Then there exists $n \in \mathbb{N}$ such that

$$
R^{i} \pi_{*}\left(L^{a} \otimes M^{b}\right)=0
$$

for all $1 \leq i \leq c$ and $a, b \in \mathbb{N}$ such that $b \geq d$ and $\max (a, b) \geq n$.

Proof. As in the proof of the previous lemma, we may assume that $S$ is affine and observe that the pair of maps to projective spaces, determined by suitable powers of $L$ and $M$, leads to the vanishing of all higher cohomology for $\min (a, b) \geq n$, for some $n \in \mathbb{N}$. By ampleness, after 
possibly increasing $n$, we obtain the vanishing for all $a, b \in \mathbb{N}$ with $b \geq n$. Now we consider a fixed $b$ with $d \leq b<n$ and $e$ with $0 \leq e<\ell$, where $\ell$ is the exponent of $M(X, L)$, and apply the hypothesis, the Leray spectral sequence, and the machinery of cohomology and base change to obtain

$$
H^{i}\left(X, L^{a \ell+e} \otimes M^{b}\right) \cong H^{i}\left(Y, \mathcal{O}_{Y}(a) \otimes \psi_{*}\left(L^{e} \otimes M^{b}\right)\right),
$$

which by ampleness vanishes for sufficiently large $a$. This gives the result.

We recall $[29,6.10 .1]$, a locally Noetherian scheme $Y$ is said to be normally flat along a closed subscheme $F$ if $\mathcal{I}_{F}^{j} / \mathcal{I}_{F}^{j+1}$ is a locally free sheaf of $\mathcal{O}_{F}$-modules for every $j$, where $\mathcal{I}_{F}$ denotes the ideal sheaf of $F$.

Proposition A.7. Let $X$ be a projective scheme over $k$ with ample line bundle $L_{0}$ and effective Cartier divisor $E \subset X$. Suppose for some $m>$ 0 , the line bundle $\left.L_{0}\right|_{E} \otimes\left(N_{E / X}\right)^{m}$ is semiample and determines a flat contraction $\psi: E \rightarrow F$. Suppose, furthermore, $N_{E / X}^{\vee}$ is ample relative to $\psi$ and on fibers is normally generated with vanishing $H^{1}$ of all positive powers. Then $L:=L_{0}(m E)$ is semiample with exponent of $M(X, L)$ equal to exponent of $M\left(E,\left.L_{0}\right|_{E} \otimes\left(N_{E / X}\right)^{m}\right)$ and determines a contraction $X \cong B \ell_{F} Y \rightarrow Y$ of $E$ to $F$ such that $Y$ is normally flat along $F$ with normal cone

$$
\operatorname{Spec}\left(\bigoplus_{n \geq 0} \psi_{*}\left(N_{E / X}^{\vee}\right)^{n}\right) .
$$

Proof. Replacing $L_{0}$ by some power, we may suppose that $L_{0}$ is very ample, with $H^{1}\left(X, L_{0}\right)=0, m \geq 2$, and $\left.L\right|_{E}$ globally generated, with sections defining $\psi: E \rightarrow F$ as in the statement. Since the tensor product of a very ample line bundle and a globally generated line bundle is very ample [27, 4.4.8], for every $1 \leq a \leq m-1$ the line bundle

$$
\left(\left.L_{0}\right|_{E}\right)^{m-a} \otimes\left(\left.L_{0}\right|_{E} \otimes N_{E / X}^{m}\right)^{a} \cong\left(\left.L_{0}\right|_{E}\right)^{m} \otimes\left(N_{E / X}\right)^{a m}
$$

is very ample. So, $\left.L_{0}\right|_{E} \otimes\left(N_{E / X}\right)^{a}$ is ample for $1 \leq a \leq m-1$. Now we claim,

$$
H^{1}\left(E,\left(\left.L_{0}\right|_{E}\right)^{n_{1}} \otimes\left(N_{E / X}\right)^{n_{2}}\right)=0
$$

for all sufficiently large $n_{1}$ and $0 \leq n_{2}<m n_{1}$. Indeed, Lemma A.5 applied to the pairs of line bundles $\left.L_{0}\right|_{E} \otimes\left(N_{E / X}\right)^{a-1}$ and $\left.L_{0}\right|_{E} \otimes\left(N_{E / X}\right)^{a}$ for $a=1, \ldots, m-1$ takes care of the case $0 \leq n_{2} \leq(m-1) n_{1}$. We consider the pair of line bundles $\left.L\right|_{E}$ (semiample) and $M:=\left.L_{0}\right|_{E} \otimes$ $\left(N_{E / X}\right)^{m-1}$ (ample). Since the restriction of $L$ to any fiber of $\psi$ is trivial, the restriction of $M$ is isomorphic to the restriction of $N_{E / X}^{\vee}$, and by assumption $H^{1}$ of all positive powers vanish. An application of Lemma 
A.6 completes the verification of the claim. As a consequence, after again replacing $L_{0}$ by a power, we may suppose that

$$
H^{1}\left(E,\left.L_{0}\right|_{E} \otimes\left(N_{E / X}\right)^{j}\right)=0
$$

for $0 \leq j<m$.

The exact sequence

$$
\left.0 \rightarrow L_{0}((j-1) E) \rightarrow L_{0}(j E) \rightarrow L_{0}\right|_{E} \otimes\left(N_{E / X}\right)^{j} \rightarrow 0
$$

and an inductive argument lead to $H^{1}\left(X, L_{0}(j E)\right)=0$ for $0 \leq j<m$. By the vanishing for $j=m-1$ and $j=m-2$, the homomorphisms

$$
H^{0}(X, L) \rightarrow H^{0}\left(E,\left.L\right|_{E}\right) \quad \text { and } \quad H^{0}(X, L(-E)) \rightarrow H^{0}\left(E,\left.L\right|_{E} \otimes N_{E / X}^{\vee}\right)
$$

are surjective. It follows that $L$ is globally generated, and if we let $\pi: X \rightarrow Y$ denote the contraction that it determines, then the image of the restriction of $\pi$ to $E$ is a copy of $F$ in $Y$, such that the schemetheoretic pre-image of $F$ under $\pi$ is $E$. Moreover, $\pi$ induces a surjective homomorphism of sheaves

$$
\mathcal{I}_{F} / \mathcal{I}_{F}^{2} \rightarrow \psi_{*}\left(\mathcal{I}_{E} / \mathcal{I}_{E}^{2}\right)
$$

Let $\mathfrak{Y}$ denotes the formal completion of $Y$ along $F$. By the Theorem on Formal Functions in the form $[28,4.1 .5], \pi$ induces an isomorphism

$$
\mathcal{O}_{\mathfrak{Y}} \stackrel{\sim}{\rightarrow} \underset{\lim }{\longleftarrow} \pi_{*}\left(\mathcal{O}_{X} / \mathcal{I}_{E}^{n}\right) \text {. }
$$

We claim that the isomorphism (A.12) comes from isomorphisms

$$
\mathcal{O}_{Y} / \mathcal{I}_{F}^{n} \stackrel{\sim}{\rightarrow} \pi_{*}\left(\mathcal{O}_{X} / \mathcal{I}_{E}^{n}\right)
$$

for every $n$.

We start by observing, by the machinery of cohomology and base change, that the hypothesis of vanishing of $H^{1}$ of positive powers of $N_{E / X}^{\vee}$ implies $R^{1} \psi_{*}\left(\mathcal{I}_{E}^{j} / \mathcal{I}_{E}^{j+1}\right)=0$ for all $j>0$. A consequence is the surjectivity of

$$
\pi_{*}\left(\mathcal{I}_{E}^{i} / \mathcal{I}_{E}^{j+1}\right) \rightarrow \pi_{*}\left(\mathcal{I}_{E}^{i} / \mathcal{I}_{E}^{j}\right)
$$

for all $i<j$. As a consequence, in the inverse system in (A.12) the transition maps are epimorphisms, and the same holds with $\mathcal{O}_{X}$ replaced by any positive power of $\mathcal{I}_{E}$.

By Lemma A.4, the homomorphism

$$
\psi_{*}\left(\mathcal{I}_{E} / \mathcal{I}_{E}^{2}\right) \otimes \psi_{*}\left(\mathcal{I}_{E}^{j-1} / \mathcal{I}_{E}^{j}\right) \rightarrow \psi_{*}\left(\mathcal{I}_{E}^{j} / \mathcal{I}_{E}^{j+1}\right)
$$

induced by $\pi$ is surjective for all $j$. Combining this with (A.11) we have the surjectivity of

$$
\mathcal{I}_{F} / \mathcal{I}_{F}^{2} \otimes \psi_{*}\left(\mathcal{I}_{E}^{j-1} / \mathcal{I}_{E}^{j}\right) \rightarrow \psi_{*}\left(\mathcal{I}_{E}^{j} / \mathcal{I}_{E}^{j+1}\right) .
$$


The remainder of the argument follows closely the proof of Proposition A.3. If $V \subset Y$ is any affine open and $s_{1}, \ldots, s_{\ell}$ are sections that generate $\left.\mathcal{I}_{F}\right|_{V}$ as a sheaf of $\mathcal{O}_{V}$-modules, then multiplication by $s_{1}, \ldots, s_{\ell}$ induces surjective

$$
H^{0}\left(U, \mathcal{I}_{E}^{i} \cdot \mathcal{I}_{E}^{j}\right)^{\ell} \rightarrow H^{0}\left(U, \mathcal{I}_{E}^{i+1} / \mathcal{I}_{E}^{j+1}\right)
$$

for all $i<j$, where $U \subset X$ denotes the pre-image of $V$. We see this by considering exact sequences, analogous to (A.5)-(A.6), but with sections on $U$ instead of $X$.

Fixing $i$ and letting $K_{j}$ denote the kernel of (A.15), we write the corresponding short exact sequences for consecutive values $j=n-1$ and $j=n$ and connect them by homomorphisms of the form (A.14), to see that the induced homomorphism of kernels $K_{n} \rightarrow K_{n-1}$ is surjective. After applying lim we obtain a short exact sequence, which tells us that

$$
\mathcal{I}_{F} \lim _{\longleftarrow} \pi_{*}\left(\mathcal{I}_{E}^{i} / \mathcal{I}_{E}^{n}\right)=\lim _{\longleftarrow} \pi_{*}\left(\mathcal{I}_{E}^{i+1} / \mathcal{I}_{E}^{n}\right) .
$$

An inductive argument, as in the proof of Proposition A.3, shows that we have an isomorphism

$$
\mathcal{I}_{F}^{i} \mathcal{O}_{\mathfrak{Y}} \stackrel{\sim}{\rightarrow} \lim _{\longleftarrow} \pi_{*}\left(\mathcal{I}_{E}^{i} / \mathcal{I}_{E}^{n}\right),
$$

for every $i$.

The claim is now immediate from (A.12) and (A.16). The claim implies that for every $n$ we have an isomorphism

$$
\mathcal{I}_{F}^{n-1} / \mathcal{I}_{F}^{n} \stackrel{\sim}{\rightarrow} \psi_{*}\left(\mathcal{I}_{E}^{n-1} / \mathcal{I}_{E}^{n}\right) \text {. }
$$

The sheaf on the right is locally free by cohomology and base change, hence $Y$ is normally flat along $F$. The statement concerning the normal cone in the proposition is immediate from (A.17). The conclusion of the proof is exactly as in Proposition A.3.

The general result and examples are valid as well in a relative setting. If $S$ is a Noetherian scheme and $X$ is a scheme, proper over $S$, then we call a line bundle $L$ on $X$ relatively semiample if over every affine open subscheme of $S$ some positive power of $L$ is globally generated. Equivalently, there exists $m>0$ such that over every affine open subscheme $L^{m}$ is globally generated. The set of natural numbers $m$ with this property will be denoted by $M(X / S, L)$, or just $M(X, L)$ when no confusion will arise. There is, as before, a well-defined exponent of $M(X / S, L)$.

Proposition A.8. Let $X$ be a scheme with a proper morphism $\varphi: X \rightarrow$ $S$ to a Noetherian scheme $S$, relatively semiample line bundle $L$, and surjective morphism of sheaves $\varphi^{*} \mathcal{E} \rightarrow L^{m}$, for some $m>0$, and coherent sheaf $\mathcal{E}$ on $S$, thereby inducing a morphism

$$
\psi: X \rightarrow \operatorname{Proj}\left(\operatorname{Sym}^{\bullet} \mathcal{E}\right) .
$$


Let $Y=\psi(X)$, and write the Stein factorization of $\psi$ as proper morphism

$$
\pi: X \rightarrow X^{\prime}
$$

followed by finite morphism $f: X^{\prime}=\operatorname{Spec}\left(\psi_{*} \mathcal{O}_{X}\right) \rightarrow Y$. We let $\mathcal{O}_{Y}(1)$, respectively $\mathcal{O}_{X^{\prime}}(1)$ denote the restriction to $Y$, respectively the pull-back to $X^{\prime}$ of $\left.\mathcal{O}_{\operatorname{Proj}(\operatorname{Sym}} \cdot \mathcal{E}\right)(1)$, so $\pi^{*} \mathcal{O}_{X^{\prime}}(1) \cong L^{m}$. Then:

(i) The morphism $\pi: X \rightarrow X^{\prime}$ is proper and induces an isomorphism $\mathcal{O}_{X^{\prime}} \stackrel{\sim}{\rightarrow} \pi_{*} \mathcal{O}_{X}$

(ii) For all $n \gg 0$, the line bundle $\mathcal{O}_{X^{\prime}}(n)$ is relatively very ample over $S$, and for any positive integer $n$ such that $\mathcal{O}_{X^{\prime}}(n)$ is relatively very ample, the morphism $X \rightarrow \operatorname{Proj}\left(\operatorname{Sym}^{\bullet} \varphi_{*}\left(L^{m n}\right)\right)$ determined by the direct image of $L^{m n}$ factors uniquely through $X^{\prime}$, identifying $X^{\prime}$ with the image of $X$ in $\operatorname{Proj}\left(\operatorname{Sym}^{\bullet} \varphi_{*}\left(L^{\otimes n}\right)\right)$.

(iii) With the property in (ii) the scheme $X^{\prime}$, with $\pi: X \rightarrow X^{\prime}$, is determined uniquely up to canonical isomorphism, independently of the choice of $m$, coherent sheaf $\mathcal{E}$, and morphism $\varphi^{*} \mathcal{E} \rightarrow L^{m}$, and the powers of $L$ that are isomorphic to the pull-back of a line bundle from $X^{\prime}$ are precisely all multiples of the exponent of $M(X / S, L)$.

(iv) If $\varphi$ is flat and there is a positive integer $n$ such that for all $i>0$,

$$
R^{i} \varphi_{*}\left(L^{n}\right)=R^{i} \varphi_{*}\left(L^{2 n}\right)=\cdots=0,
$$

then the corresponding direct image sheaves are locally free, their formation commutes with arbitrary base change $S^{\prime} \rightarrow S$, when $S^{\prime}$ is Noetherian the pull-back of $L$ to $S^{\prime} \times{ }_{S} X$ determines the contraction $S^{\prime} \times{ }_{S} X \rightarrow S^{\prime} \times{ }_{S} X^{\prime}$, and $X^{\prime}$ is flat over $S$.

Proof. As in Proposition A.1, (i) recalls a basic property of the Stein factorization. For (ii), we have as before the first statment by standard facts on very ample line bundles. Letting $\rho: X^{\prime} \rightarrow S$ denote the structure morphism, using the isomorphism $\mathcal{O}_{X^{\prime}} \cong \pi_{*} \mathcal{O}_{X}$ we have $\varphi_{*}\left(L^{\otimes n}\right) \cong \rho_{*}\left(\mathcal{O}_{X^{\prime}}(n)\right)$, and this determes compatible morphisms to its projectivization from $X$ and from $X^{\prime}$, the latter a closed immersion. For (iii) the argument is as in Proposition A.1. The statements concerning the direct image sheaves in (iv) follow from cohomology and base change, and the flatness from

$$
X^{\prime} \cong \operatorname{Proj}\left(\mathcal{O}_{S} \oplus \varphi_{*}\left(L^{\otimes n}\right) \oplus \varphi_{*}\left(L^{\otimes 2 n}\right) \oplus \ldots\right) .
$$

The remaining assertion follows from these facts, using that some multiple of $n$ satisfies the condition in (ii) both for $X$ and for $S^{\prime} \times{ }_{S} X$.

Proposition A.9. Let $S$ be a Noetherian scheme, and let $X$ be a projective scheme over $S$ (resp. a projective flat scheme over $S$ ) with relatively 
ample line bundle $L_{0}$ and effective Cartier divisor $E \subset X$. Suppose for some $m>0$, the line bundle $\left.L_{0}\right|_{E} \otimes\left(N_{E / X}\right)^{m}$ over affine open subsets of $S$ is semiample and determines a flat contraction $\psi: E \rightarrow F$. Suppose, furthermore, $N_{E / X}^{\vee}$ is ample relative to $\psi$ and on fibers is normally generated with vanishing $H^{1}$ of all positive powers (resp. vanishing $H^{i}$ for all $i>0$ of all nonnegative powers). Then $L:=L_{0}(m E)$ is semiample with exponent of $M(X, L)$ equal to exponent of $M\left(E,\left.L_{0}\right|_{E} \otimes\left(N_{E / X}\right)^{m}\right)$ over affine open subsets of $S$ and determines a contraction $X \cong B \ell_{F} Y \rightarrow Y$ of $E$ to $F$ (resp. a contraction $X \cong B \ell_{F} Y \rightarrow Y$ of $E$ to $F$ with $Y$ flat over $S$, whose formation commutes with arbitrary base change $S^{\prime} \rightarrow S$ with $S^{\prime}$ Noetherian) such that $Y$ is normally flat along $F$ with normal cone

$$
\operatorname{Spec}\left(\bigoplus_{n \geq 0} \psi_{*}\left(N_{E / X}^{\vee}\right)^{n}\right)
$$

Proof. We may suppose $S$ is affine. Now we argue as in the proof of Proposition A.7, relative over $S$. In the flat case with the stronger hypothesis of cohomology vanishing we have (A.10) also for $j=m$, also with $L_{0}$ replaced by $L_{0}^{n}$ for any $n>0$ and all $0 \leq j \leq m n$, and also with any $H^{i}(i>0)$ in place of $H^{1}$. Hence $H^{i}\left(X, L^{n}\right)=0$ for all $i>0$ and $n>0$. Proposition A.8(iii) gives us the desired conclusion.

Remark A.10. Proposition A.9 is a strict generalization of Proposition A.7, which in turn generalizes Proposition A.3; the inclusion of all three results serves expository purposes only.

Remark A.11. In Proposition A.3, respectively A.7 and A.9, the morphism $\pi: X \rightarrow Y$ induces by pullback and direct image an equivalence of categories between locally free coherent sheaves on $Y$ and locally free coherent sheaves on $X$ whose restriction to $E$ is free, respectively isomorphic to $\psi^{*}$ of a locally free coherent sheaf on $F$. That $\pi_{*}$ of such a sheaf $\mathcal{F}$ is locally free follows from the Theorem on Formal Functions and observations concerning $H^{0}\left(X, \mathcal{I}_{E}^{i} \mathcal{F} / \mathcal{I}_{E}^{j} \mathcal{F}\right)$, respectively $H^{0}\left(U, \mathcal{I}_{E}^{i} \mathcal{F} / \mathcal{I}_{E}^{j} \mathcal{F}\right)$, analogous to those in (A.3)-(A.4), respectively (A.14)-(A.15).

Remark A.12. In Proposition A.9 (without the assumption that $X \rightarrow S$ is flat and stronger cohomology vanishing from the statement), $\pi: X \rightarrow Y$ is characterized by the following universal property: for any $S$-scheme $Z$, S-morphisms $Y \rightarrow Z$ are by composition with $\pi$ in bijection with $S$-morphisms $X \rightarrow Z$ whose restriction to $E$ factors through $F$ (by $\psi$ ). (Such a factorization, if it exists, is unique by [32, VIII.5.1(a)].) Indeed, such an $S$-morphism $X \rightarrow Z$ induces a unique compatible map $Y \rightarrow Z$ on the level of sets, continuous since $\pi$ is proper and surjective and hence a subset $U \subset Y$ is open if and only if $\pi^{-1}(U) \subset X$ is open, and with $\pi$ 
being a contraction, there is a unique compatible morphism of structure sheaves. In fact, we may allow $Z$ to be an algebraic space; then we need the observation that given compatible separated étale morphisms of schemes $X^{\prime} \rightarrow X, E^{\prime} \rightarrow E$, and $F^{\prime} \rightarrow F$, the functor on $Y$-schemes that sends $\widetilde{Y} \rightarrow Y$, determining $\widetilde{X} \rightarrow X, \widetilde{E} \rightarrow E$, and $\widetilde{F} \rightarrow F$ by base change, to the set of compatible lifts $\widetilde{X} \rightarrow X^{\prime}, \widetilde{E} \rightarrow E^{\prime}$, and $\widetilde{F} \rightarrow F^{\prime}$, is representable by a scheme $Y^{\prime}$, separated and étale over $Y$. Already the functor sending $\tilde{Y} \rightarrow Y$ to the set of lifts $\widetilde{F} \rightarrow F^{\prime}$ is representable by an algebraic space, étale over $Y[53, \S 1.1][5, \S 5.1]$, so we may suppose $F^{\prime}=F\left(\right.$ and $\left.E^{\prime}=E\right)$. Then the observation follows from a general result of Artin on contractions [6, Thm. 3.1] and the property of separated étale morphisms of algebraic spaces that if the target is a scheme then so is the source [39, Cor. II.6.17]. The universal property in this level of generality compares nicely with earlier results, e.g., in [49].

\section{REFERENCES}

[1] D. Abramovich, T. Graber, and A. Vistoli. Gromov-Witten theory of DeligneMumford stacks. Amer. J. Math., 130(5):1337-1398, 2008.

[2] D. Abramovich, M. Olsson, and A. Vistoli. Tame stacks in positive characteristic. Ann. Inst. Fourier (Grenoble), 58(4):1057-1091, 2008.

[3] J. Alper. Good moduli spaces for Artin stacks. Ann. Inst. Fourier (Grenoble), 63(6):2349-2402, 2013.

[4] J. Alper, J. Hall, and D. Rydh. A Luna étale slice theorem for algebraic stacks, 2015. arXiv: 1504.06467.

[5] J. Alper and A. Kresch. Equivariant versal deformations of semistable curves. Michigan Math. J., 65(2):227-250, 2016.

[6] M. Artin. Algebraization of formal moduli. II. Existence of modifications. Ann. of Math. (2), 91:88-135, 1970.

[7] M. Artin. Brauer-Severi varieties. In Brauer groups in ring theory and algebraic geometry (Wilrijk, 1981), volume 917 of Lecture Notes in Math., pages 194-210. Springer, Berlin-New York, 1982.

[8] M. Artin. Left ideals in maximal orders. In Brauer groups in ring theory and algebraic geometry (Wilrijk, 1981), volume 917 of Lecture Notes in Math., pages 182-193. Springer, Berlin-New York, 1982.

[9] M. Artin. Local structure of maximal orders on surfaces. In Brauer groups in ring theory and algebraic geometry (Wilrijk, 1981), volume 917 of Lecture Notes in Math., pages 146-181. Springer, Berlin-New York, 1982.

[10] M. Artin, A. Grothendieck, and J. L. Verdier. Théorie des topos et cohomologie étale des schémas (SGA 4), tome 3, volume 305 of Lecture Notes in Math. Springer, Berlin-New York, 1973.

[11] M. Artin and D. Mumford. Some elementary examples of unirational varieties which are not rational. Proc. London Math. Soc. (3), 25:75-95, 1972.

[12] A. Auel, M. Bernardara, and M. Bolognesi. Fibrations in complete intersections of quadrics, Clifford algebras, derived categories, and rationality problems. $J$. Math. Pures Appl. (9), 102(1):249-291, 2014. 
[13] A. Beauville. A very general sextic double solid is not stably rational. Bull. Lond. Math. Soc., 48(2):321-324, 2016.

[14] D. Bergh. Functorial destackification of tame stacks with abelian stabilisers. Compos. Math., 153(6):1257-1315, 2017.

[15] S. Bloch and A. Ogus. Gersten's conjecture and the homology of schemes. Ann. Sci. École Norm. Sup. (4), 7:181-201 (1975), 1974.

[16] C. Böhning and H.-C. Graf von Bothmer. On stable rationality of some conic bundles and moduli spaces of Prym curves, 2016. arXiv:1605.03029, to appear in Comm. Math. Helvetici.

[17] J. W. Bruce and C. T. C. Wall. On the classification of cubic surfaces. J. London Math. Soc. (2), 19(2):245-256, 1979.

[18] C. Cadman. Using stacks to impose tangency conditions on curves. Amer. J. Math., 129(2):405-427, 2007.

[19] J.-L. Colliot-Thélène. Birational invariants, purity and the Gersten conjecture. In $K$-theory and algebraic geometry: connections with quadratic forms and division algebras (Santa Barbara, CA, 1992), volume 58 of Proc. Sympos. Pure Math., pages 1-64. Amer. Math. Soc., Providence, RI, 1995.

[20] J.-L. Colliot-Thélène and M. Ojanguren. Variétés unirationnelles non rationnelles: au-delà de l'exemple d'Artin et Mumford. Invent. Math., 97(1):141$158,1989$.

[21] J.-L. Colliot-Thélène and A. Pirutka. Hypersurfaces quartiques de dimension 3: non-rationalité stable. Ann. Sci. École Norm. Sup. (4), 49(2):371-397, 2016.

[22] V. Cossart, U. Jannsen, and S. Saito. Canonical embedded and nonembedded resolution of singularities for excellent two-dimensional schemes, 2009. arXiv:0905.2191.

[23] A. J. de Jong. The period-index problem for the Brauer group of an algebraic surface. Duke Math. J., 123(1):71-94, 2004.

[24] P. Deligne and D. Mumford. The irreducibility of the space of curves of given genus. Inst. Hautes Études Sci. Publ. Math., 36:75-109, 1969.

[25] D. Edidin, B. Hassett, A. Kresch, and A. Vistoli. Brauer groups and quotient stacks. Amer. J. Math., 123(4):761-777, 2001.

[26] B. H. Gross and J. Harris. On some geometric constructions related to theta characteristics. In Contributions to automorphic forms, geometry, and number theory, pages 279-311. Johns Hopkins Univ. Press, Baltimore, MD, 2004.

[27] A. Grothendieck. Éléments de géométrie algébrique. II. Étude globale élémentaire de quelques classes de morphismes. Inst. Hautes Études Sci. Publ. Math., 8, 1961.

[28] A. Grothendieck. Éléments de géométrie algébrique. III. Étude cohomologique des faisceaux cohérents. Inst. Hautes Études Sci. Publ. Math., 11, 17, 1961-63.

[29] A. Grothendieck. Éléments de géométrie algébrique. IV. Étude locale des schémas et des morphismes de schémas. Inst. Hautes Études Sci. Publ. Math., 20, 24, 28, $32,1964-67$.

[30] A. Grothendieck. Le groupe de Brauer. I-III. In Dix exposés sur la cohomologie des schémas, volume 3 of Adv. Stud. Pure Math., pages 46-188. North-Holland, Amsterdam, 1968.

[31] A. Grothendieck. Techniques de construction et théorèmes d'existence en géométrie algébrique. IV. Les schémas de Hilbert. In Séminaire Bourbaki, Vol. 6, pages Exp. No. 221, 249-276. Soc. Math. France, Paris, 1995. 
[32] A. Grothendieck. Revêtements étales et groupe fondamental (SGA 1), volume 3 of Documents Mathématiques. Société Mathématique de France, Paris, 2003.

[33] R. Hartshorne. Stable reflexive sheaves. Math. Ann., 254(2):121-176, 1980.

[34] B. Hassett, A. Kresch, and Yu. Tschinkel. Stable rationality and conic bundles. Math. Ann., 365(3-4):1201-1217, 2016.

[35] B. Hassett and Yu. Tschinkel. On stable rationality of Fano threefolds and del Pezzo fibrations, 2016. arXiv: 1601.07074, to appear in J. Reine Angew. Math.

[36] H. Hironaka. Smoothing of algebraic cycles of small dimensions. Amer. J. Math., 90:1-54, 1968.

[37] Y. Kawamata, K. Matsuda, and K. Matsuki. Introduction to the minimal model problem. In Algebraic geometry, Sendai, 1985, volume 10 of Adv. Stud. Pure Math., pages 283-360. North-Holland, Amsterdam, 1987.

[38] S. Keel and S. Mori. Quotients by groupoids. Ann. of Math. (2), 145(1):193-213, 1997.

[39] D. Knutson. Algebraic spaces. Lecture Notes in Mathematics, Vol. 203. SpringerVerlag, Berlin-New York, 1971.

[40] M. Kontsevich and Yu. Tschinkel. Specialization of birational types, 2017. arXiv: 1708.05699.

[41] A. Kresch. Flattening stratification and the stack of partial stabilizations of prestable curves. Bull. Lond. Math. Soc., 45(1):93-102, 2013.

[42] I. Krylov and T. Okada. Stable rationality of del Pezzo fibrations of low degree over projective spaces, 2017. arXiv:1701.08372.

[43] G. Laumon and L. Moret-Bailly. Champs algébriques, volume 39 of Ergebnisse der Mathematik und ihrer Grenzgebiete. 3. Folge. Springer-Verlag, Berlin, 2000.

[44] R. Lazarsfeld. Positivity in algebraic geometry. I, volume 48 of Ergebnisse der Mathematik und ihrer Grenzgebiete. 3. Folge. Springer-Verlag, Berlin, 2004.

[45] M. Lieblich. Twisted sheaves and the period-index problem. Compos. Math., 144(1):1-31, 2008.

[46] M. Lieblich. Period and index in the Brauer group of an arithmetic surface. $J$. Reine Angew. Math., 659:1-41, 2011. With an appendix by Daniel Krashen.

[47] T. Maeda. On standard projective plane bundles. J. Algebra, 197(1):14-48, 1997.

[48] K. Matsuki and M. Olsson. Kawamata-Viehweg vanishing as Kodaira vanishing for stacks. Math. Res. Lett., 12(2-3):207-217, 2005.

[49] J. Mazur. Conditions for the existence of contractions in the category of algebraic spaces. Trans. Amer. Math. Soc., 209:259-265, 1975.

[50] J. S. Milne. Étale cohomology, volume 33 of Princeton Mathematical Series. Princeton University Press, Princeton, N.J., 1980.

[51] S. Mori. Threefolds whose canonical bundles are not numerically effective. Ann. of Math. (2), 116(1):133-176, 1982.

[52] D. Mumford. Varieties defined by quadratic equations. In Questions on Algebraic Varieties (C.I.M.E., III Ciclo, Varenna, 1969), pages 29-100. Edizioni Cremonese, Rome, 1970.

[53] A. M. Mustaţă and A. Mustaţă. The structure of a local embedding and Chern classes of weighted blow-ups. J. Eur. Math. Soc., 14(6):1739-1794, 2012.

[54] J. Nicaise and E. Shinder. The motivic nearby fiber and degeneration of stable rationality, 2017. arXiv: 1708.02790.

[55] J. Oesinghaus. Conic bundles and iterated root stacks, 2017. In preparation. 
[56] M. Olsson and J. Starr. Quot functors for Deligne-Mumford stacks. Comm. Algebra, 31(8):4069-4096, 2003. Special issue in honor of Steven L. Kleiman.

[57] D. Rydh. Étale dévissage, descent and pushouts of stacks. J. Algebra, 331:194223, 2011.

[58] D. Rydh. Existence and properties of geometric quotients. J. Algebraic Geom., 22(4):629-669, 2013.

[59] D. J. Saltman. Lectures on division algebras, volume 94 of CBMS Regional Conference Series in Mathematics. Published by American Mathematical Society, Providence, RI; on behalf of Conference Board of the Mathematical Sciences, Washington, DC, 1999.

[60] V. G. Sarkisov. On conic bundle structures. Izv. Akad. Nauk SSSR Ser. Mat., 46(2):371-408, 432, 1982.

[61] M. G. Smith and J. S. Wilson. On subgroups of finite index in compact Hausdorff groups. Arch. Math. (Basel), 80(2):123-129, 2003.

[62] K.-O. Stöhr and J. F. Voloch. Weierstrass points and curves over finite fields. Proc. London Math. Soc. (3), 52(1):1-19, 1986.

[63] B. Totaro. Hypersurfaces that are not stably rational. J. Amer. Math. Soc., 29(3):883-891, 2016.

[64] C. Voisin. Unirational threefolds with no universal codimension 2 cycle. Invent. Math., 201(1):207-237, 2015.

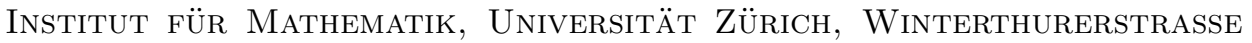
190, CH-8057 ZÜRICH, SwitzerLAND

E-mail address: andrew.kresch@math.uzh.ch

Courant Institute, 251 Mercer Street, New York, NY 10012, USA

Simons Foundation, 160 Fifth Av., New York, NY 10010, USA

E-mail address: tschinkel@cims.nyu.edu 